\title{
Geographical distribution of hyperuricemia in mainland China: a comprehensive systematic review and meta-analysis
}

Jiayun Huang ${ }^{1 \dagger}$, Zheng Feei Ma ${ }^{1,2^{*+}} \mathbb{D}$, Yutong Zhang ${ }^{3}$, Zhongxiao Wan ${ }^{4}$, Yeshan $\mathrm{Li}^{5}$, Hang Zhou ${ }^{6,7}$, Anna Chu ${ }^{8}$ and Yeong Yeh Lee ${ }^{2,9,10}$

\begin{abstract}
Background: Fructose plays an important role in the complex metabolism of uric acid in the human body. However, high blood uric acid concentration, known as hyperuricemia, is the main risk factor for development of gout. Therefore, we conducted an updated meta-analysis on the prevalence and geographical distribution of hyperuricemia among the general population in mainland China using systematic literature search.

Methods: Five electronic databases were used to search for relevant articles published until 2019. All calculations were conducted using the Comprehensive Meta-Analysis (CMA) software. We included 108 eligible articles (172 studies by sex, 95 studies by regions, and 107 studies by study type) and an overall sample size of > 808,505 participants.

Results: The pooled prevalence of hyperuricemia among the general population in mainland China was 17.4\% (95\% Cl: 15.8-19.1\%). Our subgroup analysis indicated that the pooled prevalence by regions ranged from 15.5 to $24.6 \%$. Those living Northeast region and being males had the highest prevalence $(P<0.001)$. In addition, some provinces in South Central, East and Northeast regions reported a high prevalence (> 20\%), particularly in males. An increasing prevalence was reported since 2005-2009 until 2015-2019. No publication of bias was observed as indicated by a symmetrical funnel plot and Begg and Mazumdar rank correlation ( $P=0.392)$.

Conclusion: Prevalence of hyperuricemia is increasing in China, and future studies should investigate the association between the prevalence of hyperuricemia and its risk factors in order to tackle the issue, particularly among the vulnerable groups. Also, our study was the first comprehensive study to investigate the overall prevalence of hyperuricemia in mainland China covering the six different regions.
\end{abstract}

Keywords: Uric acid, Hyperuricemia, Gout, China, Urbanisation

\section{Background}

High blood uric acid concentration, known as hyperuricemia, is the main risk factor for development of gout $[1,2]$. Uric acid is a terminal metabolite of human purine compounds, which is slightly soluble in water and

\footnotetext{
* Correspondence: zhengfeeima@gmail.com

† Jiayun Huang and Zheng Feei Ma contributed equally to this work.

'Department of Health and Environmental Sciences, Xi'an Jiaotong-Liverpool

University, Suzhou 215123, Jiangsu, China

${ }^{2}$ School of Medical Sciences, Universiti Sains Malaysia, 16150 Kota Bharu,

Kelantan, Malaysia

Full list of author information is available at the end of the article
}

easy to form crystals $[3,4]$. When uric acid increases to a certain threshold level in the human body, it is considered hyperuricemia [5].

The body has $\sim 1200 \mathrm{mg}$ and $\sim 600 \mathrm{mg}$ total body pool of exchangeable uric acid in males and females, respectively [6]. There are about $600 \mathrm{mg}$ uric acid that are produced every day, and another $600 \mathrm{mg}$ uric acid are excreted, resulting in a balanced state [7]. A disturbed state of purine metabolism can cause a variety of disorders, such as hyperuricemia, chronic gout, joint deformation and renal failure [3]. Among them, hyperuricemia has received increasing attention in recent decades

(c) The Author(s). 2020 Open Access This article is licensed under a Creative Commons Attribution 4.0 International License, which permits use, sharing, adaptation, distribution and reproduction in any medium or format, as long as you give appropriate credit to the original author(s) and the source, provide a link to the Creative Commons licence, and indicate if changes were made. The images or other third party material in this article are included in the article's Creative Commons licence, unless indicated otherwise in a credit line to the material. If material is not included in the article's Creative Commons licence and your intended use is not permitted by statutory regulation or exceeds the permitted use, you will need to obtain permission directly from the copyright holder. To view a copy of this licence, visit http://creativecommons.org/licenses/by/4.0/. 
because of its increasing global trends and risk of associated metabolic diseases. The prevalence of hyperuricemia can be influenced by several factors, including genetics, gender, age, lifestyle, diet, medication and economic development. For example, a higher prevalence is usually reported in the economically developed regions [8].

In addition, higher uric acid concentration is associated with increased risk of hospitalization, chronic kidney disease and cardiovascular disease (CVD), which can result in higher total medical costs and hospitalisation costs per patient. For example, the mean annual healthcare costs in Italy for hyperuricemic patients ranged from $€ 2752$ to $€ 4607$ [5]. Elderly patients with hyperuricemia in China are at risk of gout attacks caused by iatric problems, which may bring about complications such as deep vein thrombosis (DVT) and a prolonged hospital stay [9]. Therefore, this does not only increase the cost of medical treatment for patients, but also increase the cost of treatment for hospitals.

There are many observational studies on the prevalence of hyperuricemia, however most of them were focused on specific populations such as children from a region of mainland China. In addition, there are only two meta-analyses in the past that have examined the prevalence of hyperuricemia in mainland China; both with limitations $[10,11]$. The first meta-analysis was conducted in 2011 with 59 articles [10] and the second one was in 2015 with 44 articles [11]; both did not have comprehensive coverage of the whole of China (for example, the former one did not include Inner Mongolia, while the latter one did not include Ningxia and Qinghai). Since China is the world's most populous country with about 1.4 billion (i.e. $18.4 \%$ of the world population), updating the epidemiology of hyperuricemia can help to fill the gap in public health research and policy. To date, there have been no published English articles that have extensively reviewed the prevalence of hyperuricemia in mainland China until December 2019. Therefore, the aim of our study was to conduct a comprehensive review and quantitative meta-analysis on the prevalence of hyperuricemia in mainland China over the past two decades. In addition, analyses were also performed to provide a more detailed and updated epidemiological distribution of hyperuricemia by comparing different regions in mainland China.

\section{Methods}

\section{Search strategy}

A systematic literature search from January 1995 to December 2019 was conducted for articles published in Chinese language from the following electronic databases: Wanfang Data, Shanghai Science and Technology Innovation Resources Center (SSTIR), China National
Knowledge Infrastructure (CNKI) and Chinese Scientific Journals Fulltext Database (CQVIP). Keywords used in the database search included: "hyperuricemia" OR "high uric acid" OR "uric acid" OR "gout" AND "Chinese" OR "China" OR the name of the provinces in China. Database search results were entered into EndNote X8.2 file (Clarivate Analytics, New York, USA). The current systematic review and meta-analysis was conducted according to the Preferred Reporting Items for Systematic Reviews and Meta-Analyses (PRISMA) guidelines [12] (Fig. 1). The protocol of the systematic review and metaanalysis was registered at PROSPERO, as CRD42019141243, which is an international database of prospectively registered systematic reviews in health and social care. Since our systematic review and metaanalysis used data from published articles, there are no requirements for us to apply for the ethics approval. However, all human studies included in our systematic review and meta-analysis have been reviewed by the appropriate ethics committee in their institutions and have therefore been performed in accordance with the ethical standards laid down in an appropriate version of the WMA Declaration of Helsinki-Ethical Principles for Medical Research Involving Human Subject.

\section{Study selection}

Studies were deemed to be eligible if they met the following criteria: (1) cross-sectional, cohort or casecontrol studies that were conducted in non-pregnant adults living in mainland China; (2) prevalence of hyperuricemia and sample size were reported; (3) detailed diagnostic criteria were included; and (4) full text of the article was able to be retrieved. Studies were excluded if they were: review articles and/or meta-analyses and inclusion of terminally ill or pregnant adults as participants.

\section{Quality assessment}

The quality of eligible studies was independently assessed by two authors (J. H. and Z. F. M.) using a modified version of Newcastle-Ottawa Scale (NOS). When there were disagreements between the authors, they were resolved by discussion.

\section{Data extraction}

For all eligible studies, the information about the authors, publication year, study design, age, sex, province, cases of hyperuricemia, total sample size, prevalence of hyperuricemia and cut-offs used for the determination of hyperuricemia was extracted. The corresponding authors of eligible studies were also contacted for obtaining the missing data in their articles. 


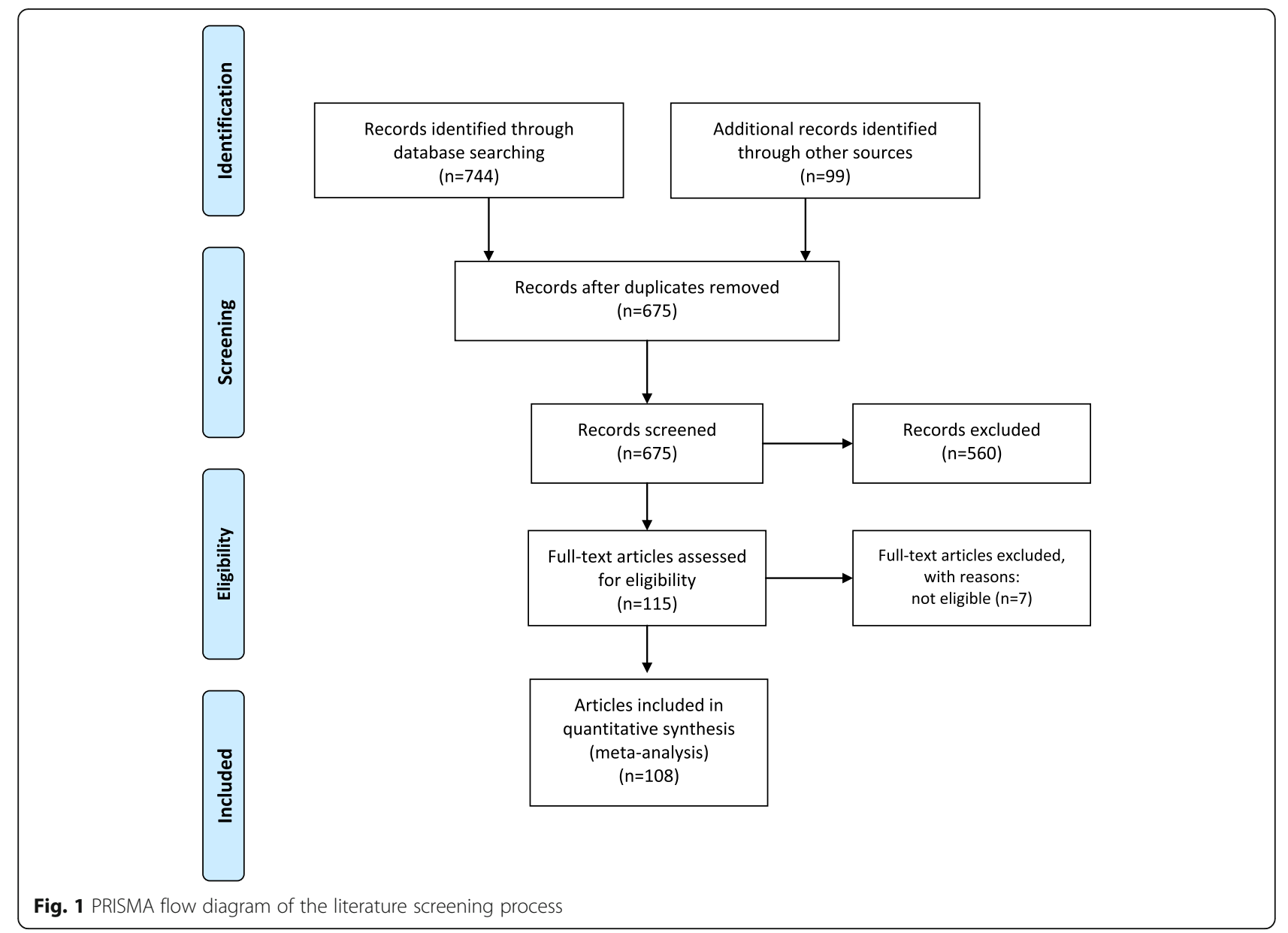

\section{Statistical analysis}

Meta-analysis was performed using the Comprehensive Meta-Analysis (CMA) software (V2.0, Biostat, Englewood, New Jersey). Random-effects models were used to estimate the pooled prevalence of hyperuricemia and 95\% confidence intervals (CI) due to the large variation of study design among the included studies. Subgroup analyses were performed by province, study design, sex and study period. Heterogeneity tests were determined using the Q-test $(P<0.10)$ and $\mathrm{I}^{2}$ statistic $(>75 \%)[13]$. Potential publication bias was assessed by the funnel plots and Begg and Mazumdar rank correlation $(P<$ $0.05)$. The one-study-removed sensitivity analysis was performed to determine the possible causes of heterogeneity between the studies.

\section{Results}

\section{Characteristics of the included studies}

A total of 108 articles were identified after screening for relevancy and duplicates (Fig. 1). Table 1 shows a detailed description of the included studies in the systematic review and meta-analysis [10-12, 14-123]. All included studies were published between 1999 and 2019 and together comprised $>808,505$ participants. Of the 108 articles, there were 172 studies by sex, 95 studies by regions, and 107 studies by study type (Table 2 ).

\section{Pooled prevalence of hyperuricemia}

The pooled estimate of prevalence in the general population was 0.174 (95\%CI: 0.158-0.191) (Fig. 2), which suggested that $17.4 \%$ of the population in mainland China had hyperuricemia.

\section{Subgroup analysis}

The prevalence of hyperuricemia was analysed in subgroups, which were categorised according to the following categories: provinces/municipalities/autonomous regions, regions (northeast, northwest, north, southwest, south central and east), sex, study type and year.

The pooled prevalence of hyperuricemia by regions ranged from 15.5 to $24.6 \%$. The pooled prevalence in Northeast region was the highest $(24.6 \%)$, followed by South Central (20.7\%), East (17.3\%), North (17.4\%), Southwest (15.8\%), and Northwest (15.5\%) (Table 2). In terms of gender distribution, the pooled prevalence of hyperuricemia in males was significantly higher than 
Table 1 Characteristics of the included studies in the systematic review and meta-analysis

\begin{tabular}{|c|c|c|c|c|c|c|c|c|c|c|}
\hline No. & Study & $\begin{array}{l}\text { Study } \\
\text { type }\end{array}$ & $\begin{array}{l}\text { Provinces (cities)/ } \\
\text { municipalities/ } \\
\text { autonomous regions }\end{array}$ & Region & $\begin{array}{l}\text { Age } \\
\text { (years) }^{c}\end{array}$ & Case & $\begin{array}{l}\text { Sample } \\
\text { size }\end{array}$ & $\begin{array}{l}\text { Prevalence } \\
(\%)\end{array}$ & Diagnostic cut-offs & Gender \\
\hline \multirow[t]{3}{*}{1} & \multirow{3}{*}{$\begin{array}{l}\text { Ma, Chen \& Li } \\
\text { (1999) [14] }\end{array}$} & \multirow[t]{3}{*}{ CS } & \multirow[t]{3}{*}{ Guangdong } & \multirow{3}{*}{$\begin{array}{l}\text { South } \\
\text { Central }\end{array}$} & \multirow[t]{3}{*}{$55-82$} & 452 & 2041 & 22.1 & $>420 \mu \mathrm{mol} / \mathrm{L}$ & Both \\
\hline & & & & & & 364 & 1696 & 21.5 & $>420 \mu \mathrm{mol} / \mathrm{L}$ & Male \\
\hline & & & & & & 88 & 345 & 25.5 & $>420 \mu \mathrm{mol} / \mathrm{L}$ & Female \\
\hline \multirow[t]{3}{*}{2} & \multirow{3}{*}{$\begin{array}{l}\text { Shao et al. } \\
\text { (2003) [15] }\end{array}$} & \multirow[t]{3}{*}{ CS } & \multirow[t]{3}{*}{ Nanjing } & \multirow[t]{3}{*}{ East } & \multirow[t]{3}{*}{$\geq 18$} & 1038 & 7778 & 13.3 & NS & Both \\
\hline & & & & & & 688 & 3790 & 17.6 & $\geq 417 \mu \mathrm{mol} / \mathrm{L}$ & Male \\
\hline & & & & & & 370 & 3988 & 9.3 & $\geq 357 \mu \mathrm{mol} / \mathrm{L}$ & Female \\
\hline \multirow[t]{3}{*}{3} & \multirow{3}{*}{$\begin{array}{l}\text { Chen et al. } \\
\text { (2004) [16] }\end{array}$} & \multirow[t]{3}{*}{ CC } & \multirow[t]{3}{*}{ Anhui } & \multirow[t]{3}{*}{ East } & \multirow[t]{3}{*}{$45 \pm 12$} & 105 & 430 & 24.4 & NS & Both \\
\hline & & & & & & 70 & 227 & 30.8 & $>420 \mu \mathrm{mol} / \mathrm{L}$ & Male \\
\hline & & & & & & 35 & 203 & 17.2 & $>360 \mu \mathrm{mol} / \mathrm{L}$ & Female \\
\hline \multirow[t]{3}{*}{4} & \multirow{3}{*}{$\begin{array}{l}\text { Wu et al. (2005) } \\
\text { [17] }\end{array}$} & \multirow[t]{3}{*}{ CS } & \multirow{3}{*}{$\begin{array}{l}\text { Guangzhou, } \\
\text { Guangdong }\end{array}$} & \multirow{3}{*}{$\begin{array}{l}\text { South } \\
\text { Central }\end{array}$} & $>55$ & 197 & 642 & 30.7 & NS & Both \\
\hline & & & & & & 46 & 152 & 30.3 & $>420 \mu \mathrm{mol} / \mathrm{L}$ & Male \\
\hline & & & & & & 151 & 490 & 30.8 & $>350 \mu \mathrm{mol} / \mathrm{L}$ & Female \\
\hline 5 & Yang et al. & CS & Shandong & East & $18-54$ & 537 & 8640 & 6.2 & NS & Both \\
\hline & (2005) [18] & & & & & 459 & 6289 & 7.3 & $\geq 416 \mu \mathrm{mol} / \mathrm{L}$ & Male \\
\hline & & & & & & 78 & 2351 & 3.3 & $\geq 357 \mu \mathrm{mol} / \mathrm{L}$ & Female \\
\hline 6 & $\begin{array}{l}\text { Wang et al. } \\
\text { (2006) [19] }\end{array}$ & CS & Shandong & East & $20-80$ & 269 & 2605 & 10.3 & $>350 \mu \mathrm{mol} / \mathrm{L}$ & Female \\
\hline 7 & Li et al. (2008) & $\mathrm{CH}$ & China $^{a}$ & $N A^{a}$ & $45-54$ & 10 & 274 & 3.6 & NS & Both \\
\hline & & & & & & 5 & 90 & 5.6 & $\geq 416 \mu \mathrm{mol} / \mathrm{L}$ & Male \\
\hline & & & & & & 5 & 184 & 2.7 & $\geq 356 \mu \mathrm{mol} / \mathrm{L}$ & Female \\
\hline & & & & & $55-64$ & 18 & 307 & 5.9 & NS & Both \\
\hline & & & & & & 13 & 138 & 9.4 & $\geq 416 \mu \mathrm{mol} / \mathrm{L}$ & Male \\
\hline & & & & & & 5 & 169 & 3.0 & $\geq 356 \mu \mathrm{mol} / \mathrm{L}$ & Female \\
\hline & & & & & $65-74$ & 21 & 229 & 9.2 & NS & Both \\
\hline & & & & & & 12 & 116 & 10.3 & $\geq 416 \mu \mathrm{mol} / \mathrm{L}$ & Male \\
\hline & & & & & & 9 & 113 & 8.0 & $\geq 356 \mu \mathrm{mol} / \mathrm{L}$ & Female \\
\hline 8 & Fan & CS & Xinyang, Henan & South & $40-75$ & 738 & 5235 & 14.1 & NS & Both \\
\hline & et al. (2009) [118] & & & & & 379 & 1763 & 21.5 & $\geq 420 \mu \mathrm{mol} / \mathrm{L}$ & Male \\
\hline & & & & & & 354 & 3472 & 10.2 & $\geq 360 \mu \mathrm{mol} / \mathrm{L}$ & Female \\
\hline 9 & $\begin{array}{l}\text { Lu et al. (2010) } \\
\text { [21] }\end{array}$ & CS & Tianjin & North & $22-53$ & 19 & 151 & 12.6 & $\geq 410 \mu \mathrm{mol} / \mathrm{L}$ & Male \\
\hline 10 & Yu et al. (2010) & CS & Foshan, Guangdong & South & $20-88$ & 1117 & 7403 & 15.1 & NS & Both \\
\hline & & & & Cer & & 714 & 3581 & 19.9 & $\geq 417 \mu \mathrm{mol} / \mathrm{L}$ & Male \\
\hline & & & & & & 403 & 3822 & 10.5 & $\geq 357 \mu \mathrm{mol} / \mathrm{L}$ & Female \\
\hline 11 & Yuan et al. & CS & Guiyang & Southwest & $>60$ & 399 & 2600 & 15.3 & $\geq 420 \mu \mathrm{mol} / \mathrm{L}$ & Both \\
\hline & (2011) [23] & & & & & 227 & 1430 & 15.9 & NS & Male \\
\hline & & & & & & 172 & 1170 & 14.7 & NS & Female \\
\hline 12 & $\begin{array}{l}\text { Zhang \& } \\
\text { Zhang (2011) } \\
{[24]}\end{array}$ & CS & China $^{a}$ & $N A^{a}$ & $\geq 18$ & 427 & 5774 & 7.4 & NS & Both \\
\hline 13 & Guo et al. (2012) & CS & Taiyuan, Shanxi & Northwest & $23-87$ & 371 & 4228 & 8.8 & NS & Both \\
\hline & & & & & & 249 & 1308 & 19.0 & $\geq 420 \mu \mathrm{mol} / \mathrm{L}$ & Male \\
\hline & & & & & & 122 & 2920 & 4.2 & $\geq 420 \mu \mathrm{mol} / \mathrm{L}$ & Female \\
\hline 14 & Wang et al. & CS & Yinchuan, Ningxia & Northwest & $\geq 18$ & 926 & 5921 & 15.6 & NS & Both \\
\hline
\end{tabular}


Table 1 Characteristics of the included studies in the systematic review and meta-analysis (Continued)

\begin{tabular}{|c|c|c|c|c|c|c|c|c|c|c|}
\hline No. & Study & $\begin{array}{l}\text { Study } \\
\text { type }\end{array}$ & $\begin{array}{l}\text { Provinces (cities)/ } \\
\text { municipalities/ } \\
\text { autonomous regions }\end{array}$ & Region & $\begin{array}{l}\text { Age } \\
\text { (years) }^{c}\end{array}$ & Case & $\begin{array}{l}\text { Sample } \\
\text { size }\end{array}$ & $\begin{array}{l}\text { Prevalence } \\
(\%)\end{array}$ & Diagnostic cut-offs & Gender \\
\hline & & & & & & 1352 & 7322 & 18.5 & NS & Both \\
\hline & & & & & & 1635 & 8717 & 18.8 & NS & Both \\
\hline \multirow[t]{3}{*}{15} & \multirow{3}{*}{$\begin{array}{l}\text { Chen et al. } \\
\text { (2013) [27] }\end{array}$} & \multirow[t]{3}{*}{ CS } & \multirow[t]{3}{*}{ Guangxi } & \multirow{3}{*}{$\begin{array}{l}\text { South } \\
\text { Central }\end{array}$} & \multirow[t]{3}{*}{$\geq 18$} & 319 & 927 & 34.4 & NS & Both \\
\hline & & & & & & 157 & 419 & 30.9 & NS & Male \\
\hline & & & & & & 162 & 508 & 38.7 & NS & Female \\
\hline \multirow[t]{3}{*}{16} & \multirow{3}{*}{$\begin{array}{l}\text { Duan et al. } \\
\text { (2013) [28] }\end{array}$} & \multirow[t]{3}{*}{ CS } & \multirow[t]{3}{*}{ Xinjiang } & \multirow[t]{3}{*}{ Northwest } & \multirow[t]{3}{*}{$\geq 18$} & 261 & 2046 & 12.8 & NS & Both \\
\hline & & & & & & 228 & 823 & 27.7 & $>417 \mu \mathrm{mol} / \mathrm{L}$ & Male \\
\hline & & & & & & 33 & 1223 & 2.7 & $>357 \mu \mathrm{mol} / \mathrm{L}$ & Female \\
\hline \multirow[t]{3}{*}{17} & \multirow{3}{*}{$\begin{array}{l}\text { Li et al. (2013) } \\
\text { [29] }\end{array}$} & \multirow[t]{3}{*}{ CS } & \multirow[t]{3}{*}{ Quanzhou, Fujian } & \multirow[t]{3}{*}{ East } & \multirow[t]{3}{*}{$40-80$} & 253 & 1358 & 18.6 & NS & Both \\
\hline & & & & & & 99 & 363 & 27.3 & $\geq 416 \mu \mathrm{mol} / \mathrm{L}$ & Male \\
\hline & & & & & & 154 & 995 & 15.5 & $\geq 357 \mu \mathrm{mol} / \mathrm{L}$ & Female \\
\hline \multirow[t]{3}{*}{18} & \multirow{3}{*}{$\begin{array}{l}\text { Li \& Cao (2013) } \\
\text { [30] }\end{array}$} & \multirow[t]{3}{*}{ CS } & \multirow[t]{3}{*}{ Karamay, Xinjiang } & \multirow[t]{3}{*}{ Northwest } & $\geq 18$ & 310 & 2032 & 15.3 & NS & Both \\
\hline & & & & & & 268 & 1086 & 24.7 & NS & Male \\
\hline & & & & & & 42 & 946 & 4.4 & NS & Female \\
\hline 19 & $\begin{array}{l}\text { Lv et al. (2013) } \\
\text { [31] }\end{array}$ & CS & Yantai, Shandong & East & $31-78$ & 66 & 635 & 10.4 & $\geq 380 \mu \mathrm{mol} / \mathrm{L}$ & Both \\
\hline 20 & Su et al. (2013) & CS & Nanhai, Guangdong & South & $45-80$ & 415 & 2015 & 20.6 & NS & Both \\
\hline & & & & Central & & 271 & 1110 & 24.4 & $>420 \mu \mathrm{mol} / \mathrm{L}$ & Male \\
\hline & & & & & & 144 & 905 & 16.9 & $>357 \mu \mathrm{mol} / \mathrm{L}$ & Female \\
\hline 21 & Wang et al. & CS & Shanghai & East & $40-70$ & 58 & 1928 & 3.0 & NS & Both \\
\hline & $(2013$ & & & & & 33 & 582 & 5.7 & $>420 \mu \mathrm{mol} / \mathrm{L}$ & Male \\
\hline & & & & & & 25 & 1346 & 1.9 & $>357 \mu \mathrm{mol} / \mathrm{L}$ & Female \\
\hline 22 & Zhang, Wu \& Lv & CS & Hebei & North & $21-95$ & 693 & 3232 & 21.4 & NS & Both \\
\hline & & & & & & 446 & 1897 & 23.5 & $\geq 428 \mu \mathrm{mol} / \mathrm{L}$ & Male \\
\hline & & & & & & 247 & 1335 & 18.5 & $\geq 357 \mu \mathrm{mol} / \mathrm{L}$ & Female \\
\hline 23 & $\begin{array}{l}\text { Zhou \& He } \\
\text { (2013) [35] }\end{array}$ & $\mathrm{CH}$ & Shenyang, Liaoning & Northeast & $50-70$ & 8 & 70 & 34.8 & NS & Both \\
\hline 24 & Chen, Dai \& Lin & CS & Guangzhou, & South & $45-75$ & 603 & 1176 & 51.3 & NS & Both \\
\hline & & & & & & 341 & 612 & 55.7 & $>420 \mu \mathrm{mol} / \mathrm{L}$ & Male \\
\hline & & & & & & 262 & 564 & 46.5 & $>357 \mu \mathrm{mol} / \mathrm{L}$ & Female \\
\hline 25 & Cui et al. (2014) & CS & Hebei & North & $\geq 20$ & 1091 & 7083 & 15.4 & NS & Both \\
\hline & & & & & & 904 & 5357 & 16.9 & $\geq 417 \mu \mathrm{mol} / \mathrm{L}$ & Male \\
\hline & & & & & & 187 & 1726 & 10.8 & $\geq 357 \mu \mathrm{mol} / \mathrm{L}$ & Female \\
\hline 26 & Li, Zhao, Gao & CS & Yunnan & Southwest & $27-89$ & 367 & 2947 & 12.5 & NS & Both \\
\hline & & & & & & 303 & 1827 & 16.6 & $>420 \mu \mathrm{mol} / \mathrm{L}$ & Male \\
\hline & & & & & & 64 & 1120 & 5.7 & $>360 \mu \mathrm{mol} / \mathrm{L}$ & Female \\
\hline 27 & Lin et al. (2014) & CS & Guangdong & South & $>60$ & 190 & 1036 & 18.3 & NS & Both \\
\hline & & & & Ce & & 86 & 383 & 22.5 & $\geq 420 \mu \mathrm{mol} / \mathrm{L}$ & Male \\
\hline & & & & & & 104 & 653 & 15.9 & $\geq 420 \mu \mathrm{mol} / \mathrm{L}$ & Female \\
\hline 28 & Liu et al. (2014) & CS & Jilin & Northeast & $38 \pm 10$ & 3395 & 16,807 & 20.2 & NS & Both \\
\hline & & & & & & 2930 & 9736 & 30.1 & NS & Male \\
\hline & & & & & & 465 & 7071 & 6.6 & NS & Female \\
\hline 29 & Pan et al. (2014) & CS & Jiangsu & East & $35-70$ & 573 & 3122 & 18.4 & NS & Both \\
\hline
\end{tabular}


Table 1 Characteristics of the included studies in the systematic review and meta-analysis (Continued)

\begin{tabular}{|c|c|c|c|c|c|c|c|c|c|c|}
\hline No. & Study & $\begin{array}{l}\text { Study } \\
\text { type }\end{array}$ & $\begin{array}{l}\text { Provinces (cities)/ } \\
\text { municipalities/ } \\
\text { autonomous regions }\end{array}$ & Region & $\begin{array}{l}\text { Age } \\
\text { (years) }^{c}\end{array}$ & Case & $\begin{array}{l}\text { Sample } \\
\text { size }\end{array}$ & $\begin{array}{l}\text { Prevalence } \\
(\%)\end{array}$ & Diagnostic cut-offs & Gender \\
\hline & & & & & & 362 & 1349 & 26.8 & $\geq 420 \mu \mathrm{mol} / \mathrm{L}$ & Male \\
\hline & & & & & & 211 & 1773 & 11.9 & $\geq 380 \mu \mathrm{mol} / \mathrm{L}$ & Female \\
\hline \multirow[t]{3}{*}{30} & \multirow{3}{*}{$\begin{array}{l}\text { Song et al. } \\
\text { (2014) [42] }\end{array}$} & \multirow[t]{3}{*}{ CS } & \multirow[t]{3}{*}{ Jiangxi } & \multirow[t]{3}{*}{ East } & \multirow[t]{3}{*}{$>40$} & 795 & 3795 & 20.9 & NS & Both \\
\hline & & & & & & 488 & 1824 & 26.8 & $>420 \mu \mathrm{mol} / \mathrm{L}$ & Male \\
\hline & & & & & & 307 & 1971 & 15.6 & $>350 \mu \mathrm{mol} / \mathrm{L}$ & Female \\
\hline \multirow[t]{3}{*}{31} & \multirow{3}{*}{$\begin{array}{l}\text { Yong \& Ye } \\
(2014) \text { [43] }\end{array}$} & \multirow[t]{3}{*}{ CS } & \multirow[t]{3}{*}{ Hebei } & \multirow[t]{3}{*}{ North } & \multirow[t]{3}{*}{$\geq 18-20$} & 813 & 5269 & 15.4 & NS & Both \\
\hline & & & & & & 769 & 2717 & 28.3 & $>420 \mu \mathrm{mol} / \mathrm{L}$ & Male \\
\hline & & & & & & 44 & 2552 & 1.7 & $>350 \mu \mathrm{mol} / \mathrm{L}$ & Female \\
\hline 32 & $\begin{array}{l}\text { Zhu, Wang, Liu } \\
\text { (2014) [44] }\end{array}$ & CS & Xinjiang & Northwest & $20-93$ & 1489 & 10,025 & 14.9 & NS & Both \\
\hline \multirow[t]{3}{*}{33} & \multirow{3}{*}{$\begin{array}{l}\text { Cao, Li \& Yi } \\
\text { (2015) [45] }\end{array}$} & \multirow[t]{3}{*}{ CS } & \multirow{3}{*}{$\begin{array}{l}\text { Guangzhou, } \\
\text { Guangdong }\end{array}$} & \multirow{3}{*}{$\begin{array}{l}\text { South } \\
\text { Central }\end{array}$} & \multirow[t]{3}{*}{$20-80$} & 290 & 988 & 29.4 & NS & Both \\
\hline & & & & & & 264 & 601 & 43.9 & $>420 \mu \mathrm{mol} / \mathrm{L}$ & Male \\
\hline & & & & & & 26 & 387 & 6.7 & $>350 \mu \mathrm{mol} / \mathrm{L}$ & Female \\
\hline \multirow[t]{3}{*}{34} & \multirow{3}{*}{$\begin{array}{l}\text { Li et al. (2015a) } \\
\text { [46] }\end{array}$} & \multirow[t]{3}{*}{$\mathrm{CS}$} & \multirow[t]{3}{*}{ Gansu } & \multirow[t]{3}{*}{ Northwest } & $48 \pm 15$ & 392 & 2364 & 16.6 & NS & Both \\
\hline & & & & & & 256 & 1254 & 20.4 & $>420 \mu \mathrm{mol} / \mathrm{L}$ & Male \\
\hline & & & & & & 136 & 1110 & 12.3 & $>360 \mu \mathrm{mol} / \mathrm{L}$ & Female \\
\hline 35 & $\begin{array}{l}\text { Li et al. (2015b) } \\
\text { [47] }\end{array}$ & $\mathrm{CS}$ & Guangxi & $\begin{array}{l}\text { South } \\
\text { Central }\end{array}$ & $\geq 20$ & $\begin{array}{l}14 \\
181\end{array}$ & 51,206 & 27.7 & NS & Both \\
\hline & & & & & & $\begin{array}{l}10 \\
722\end{array}$ & 27,144 & 39.5 & $\geq 417 \mu \mathrm{mol} / \mathrm{L}$ & Male \\
\hline & & & & & & 3459 & 24,062 & 14.4 & $\geq 357 \mu \mathrm{mol} / \mathrm{L}$ & Female \\
\hline 36 & Li et al. (2015c) & CS & Dongguan, & South & $\geq 18$ & 519 & 1375 & 37.6 & NS & Both \\
\hline & & & Guangdong & Central & & 366 & 657 & 26.6 & $>420 \mu \mathrm{mol} / \mathrm{L}$ & Male \\
\hline & & & & & & 153 & 718 & 11.1 & $>350 \mu \mathrm{mol} / \mathrm{L}$ & Female \\
\hline 37 & Liu et al. (2015) & CS & Guangzhou, & South & $\geq 18$ & 1334 & 4237 & 31.5 & NS & Both \\
\hline & & & Guangdong & Central & & 859 & 2257 & 38.1 & $>420 \mu \mathrm{mol} / \mathrm{L}$ & Male \\
\hline & & & & & & 475 & 1980 & 24.0 & $>360 \mu \mathrm{mol} / \mathrm{L}$ & Female \\
\hline 38 & Lu (2015) [49] & CS & Shanghai & East & $65-85$ & 220 & 1128 & 19.5 & NS & Both \\
\hline & & & & & & 165 & 607 & 27.2 & $>420 \mu \mathrm{mol} / \mathrm{L}$ & Male \\
\hline & & & & & & 63 & 511 & 12.3 & $>350 \mu \mathrm{mol} / \mathrm{L}$ & Female \\
\hline 39 & Zhao (2015) [50] & CS & China $^{a}$ & $N A^{a}$ & $20-60$ & 4616 & 12,650 & 36.5 & NS & Both \\
\hline 40 & Zhou et al. & CS & Sichuan & Southwest & $\geq 18$ & 182 & 972 & 18.7 & NS & Both \\
\hline & & & & & & 123 & 452 & 27.2 & $\geq 420 \mu \mathrm{mol} / \mathrm{L}$ & Male \\
\hline & & & & & & 59 & 520 & 11.3 & $\geq 360 \mu \mathrm{mol} / \mathrm{L}$ & Female \\
\hline 41 & Zhou et al. & $\mathrm{CS}$ & Henan & South & $20-60$ & 1196 & 4916 & 24.3 & NS & Both \\
\hline & & & & & & 1128 & 4290 & 26.3 & $\geq 420 \mu \mathrm{mol} / \mathrm{L}$ & Male \\
\hline & & & & & & 68 & 626 & 10.9 & $\geq 357 \mu \mathrm{mol} / \mathrm{L}$ & Female \\
\hline 42 & $\begin{array}{l}\text { Guli, He \& Zhang } \\
\text { (2016) [53] }\end{array}$ & CS & Gansu & Northwest & $20-80$ & 780 & 6400 & 12.2 & $>420 \mu \mathrm{mol} / \mathrm{L}$ & Both \\
\hline 43 & $\begin{array}{l}\text { Chen \& Xing } \\
\text { (2016) [54] }\end{array}$ & CS & Beijing & North & $25-82$ & 151 & 868 & 17.4 & $\geq 416 \mu \mathrm{mol} / \mathrm{L}$ & Male \\
\hline 44 & Chen \& Zhou & CS & Zhejiang & East & $>60$ & 691 & 4160 & 16.6 & NS & Both \\
\hline & & & & & & 393 & 2182 & 18.0 & $>420 \mu \mathrm{mol} / \mathrm{L}$ & Male \\
\hline & & & & & & 298 & 1978 & 15.1 & $>360 \mu \mathrm{mol} / \mathrm{L}$ & Female \\
\hline 45 & Fan et al. (2016) & $\mathrm{CS}$ & Shanghai & East & $\geq 18$ & 5413 & 27,615 & 19.6 & NS & Both \\
\hline
\end{tabular}


Table 1 Characteristics of the included studies in the systematic review and meta-analysis (Continued)

\begin{tabular}{|c|c|c|c|c|c|c|c|c|c|c|}
\hline No. & Study & $\begin{array}{l}\text { Study } \\
\text { type }\end{array}$ & $\begin{array}{l}\text { Provinces (cities)/ } \\
\text { municipalities/ } \\
\text { autonomous regions }\end{array}$ & Region & $\begin{array}{l}\text { Age } \\
\text { (years) }\end{array}$ & Case & $\begin{array}{l}\text { Sample } \\
\text { size }\end{array}$ & $\begin{array}{l}\text { Prevalence } \\
\text { (\%) }\end{array}$ & Diagnostic cut-offs & Gender \\
\hline & {$[56]$} & & & & & 3993 & 14,104 & 28.3 & $>420 \mu \mathrm{mol} / \mathrm{L}$ & Male \\
\hline & & & & & & 1420 & 13,511 & 10.5 & $>357 \mu \mathrm{mol} / \mathrm{L}$ & Female \\
\hline \multirow[t]{3}{*}{46} & \multirow{3}{*}{$\begin{array}{l}\text { Feng et al. } \\
\text { (2016) [57] }\end{array}$} & \multirow[t]{3}{*}{ CS } & \multirow[t]{3}{*}{ Jiangsu } & \multirow[t]{3}{*}{ East } & \multirow[t]{3}{*}{$18-93$} & 219 & 1352 & 16.2 & NS & Both \\
\hline & & & & & & 129 & 609 & 21.2 & $>420 \mu \mathrm{mol} / \mathrm{L}$ & Male \\
\hline & & & & & & 90 & 743 & 12.1 & $>350 \mu \mathrm{mol} / \mathrm{L}$ & Female \\
\hline 47 & Li (2016) [58] & CS & Tianjin & North & $\geq 18$ & $\begin{array}{l}10 \\
344\end{array}$ & 77,787 & 13.3 & NS & Both \\
\hline \multirow[t]{3}{*}{48} & \multirow{3}{*}{$\begin{array}{l}\text { Li et al. (2016) } \\
\text { [59] }\end{array}$} & \multirow[t]{3}{*}{ CS } & \multirow[t]{3}{*}{ Chongqing } & \multirow[t]{3}{*}{ Southwest } & \multirow[t]{3}{*}{39} & 1596 & 26,067 & 6.1 & NS & Both \\
\hline & & & & & & 1272 & 18,139 & 7.0 & $\geq 420 \mu \mathrm{mol} / \mathrm{L}$ & Male \\
\hline & & & & & & 324 & 7928 & 4.1 & $\geq 357 \mu \mathrm{mol} / \mathrm{L}$ & Female \\
\hline \multirow[t]{3}{*}{49} & \multirow{3}{*}{$\begin{array}{l}\text { Liu et al. (2016) } \\
\text { [60] }\end{array}$} & \multirow[t]{3}{*}{ CS } & \multirow[t]{3}{*}{ Shanghai } & \multirow[t]{3}{*}{ East } & \multirow[t]{3}{*}{$\geq 18$} & 8100 & 9653 & 83.9 & NS & Both \\
\hline & & & & & & 2872 & 3550 & 81.2 & $>420 \mu \mathrm{mol} / \mathrm{L}$ & Male \\
\hline & & & & & & 5228 & 6103 & 85.9 & $>357 \mu \mathrm{mol} / \mathrm{L}$ & Female \\
\hline \multirow[t]{3}{*}{50} & \multirow{3}{*}{$\begin{array}{l}\text { Liu, Zhou \& Yin } \\
\text { (2016) [61] }\end{array}$} & \multirow[t]{3}{*}{ CS } & \multirow[t]{3}{*}{ Yunnan } & \multirow[t]{3}{*}{ Southwest } & $32-60$ & 131 & 390 & 33.6 & NS & Both \\
\hline & & & & & & 126 & 334 & 37.7 & $>420 \mu \mathrm{mol} / \mathrm{L}$ & Male \\
\hline & & & & & & 5 & 56 & 9.1 & $>360 \mu \mathrm{mol} / \mathrm{L}$ & Female \\
\hline 51 & Lu (2016) [62] & CS & Xinjiang & Northwest & $\geq 60$ & 233 & 986 & 23.6 & NS & Both \\
\hline 52 & $\begin{array}{l}\text { Pu et al. (2016) } \\
\text { [63] }\end{array}$ & CS & China $^{a}$ & $N A^{a}$ & $20-91$ & 1078 & 11,967 & 9.0 & NS & Both \\
\hline 53 & Wang (2016) [64] & CS & Hubei & South & $18-22$ & 358 & 4333 & 8.3 & NS & Both \\
\hline & & & & & & 294 & 2029 & 14.5 & $>420 \mu \mathrm{mol} / \mathrm{L}$ & Male \\
\hline & & & & & & 64 & 2304 & 2.8 & $>350 \mu \mathrm{mol} / \mathrm{L}$ & Female \\
\hline 54 & Xie et al. (2016) & CS & Beijing; Tangshan and & North & $18-60$ & 632 & 2782 & 22.7 & NS & Both \\
\hline & & & & & & 268 & 1830 & 14.6 & $>420 \mu \mathrm{mol} / \mathrm{L}$ & Male \\
\hline & & & & & & 364 & 952 & 35.1 & $>357 \mu \mathrm{mol} / \mathrm{L}$ & Female \\
\hline 55 & Yang, Wang \& & CS & Tianjin & North & $18-93$ & 1165 & 8968 & 13.0 & NS & Both \\
\hline & Wang (2016) [66] & & & & & 959 & 5449 & 17.6 & $>417 \mu \mathrm{mol} / \mathrm{L}$ & Male \\
\hline & & & & & & 206 & 3519 & 5.9 & $>357 \mu \mathrm{mol} / \mathrm{L}$ & Female \\
\hline 56 & Zhang (2016) & CS & China $^{a}$ & $N A^{a}$ & $\geq 18$ & 198 & 794 & 24.9 & $>420 \mu \mathrm{mol} / \mathrm{L}$ & Male \\
\hline & & & Eastern China ${ }^{a}$ & East & $\geq 18$ & 58 & 202 & 31.3 & $>421 \mu \mathrm{mol} / \mathrm{L}$ & Male \\
\hline 57 & $\begin{array}{l}\text { Zhao et al. } \\
\text { (2016a) [68] }\end{array}$ & CS & Lanzhou, Gansu & Northwest & $\geq 45$ & 37 & 175 & 21.1 & NS & Both \\
\hline 58 & Zhao et al. & CS & Beijing & North & $20 \pm 3$ & 1716 & 6400 & 26.8 & NS & Both \\
\hline & & & & & & 1464 & 4198 & 34.9 & $>417 \mu \mathrm{mol} / \mathrm{L}$ & Male \\
\hline & & & & & & 252 & 2202 & 11.4 & $>357 \mu \mathrm{mol} / \mathrm{L}$ & Female \\
\hline 59 & Zhao et al. & CS & Beijing & North & $20-89$ & 1086 & 6690 & 16.2 & NS & Both \\
\hline & (2016c) [70] & & & & & 785 & 3339 & 23.5 & $>417 \mu \mathrm{mol} / \mathrm{L}$ & Male \\
\hline & & & & & & 301 & 3351 & 10.0 & $>357 \mu \mathrm{mol} / \mathrm{L}$ & Female \\
\hline 60 & Feng et al. & CS & Beijing & North & range $\geq$ & 2257 & 12,335 & 18.3 & NS & Both \\
\hline & (2017) [71] & & & & & 1867 & 7681 & 24.3 & $>420 \mu \mathrm{mol} / \mathrm{L}$ & Male \\
\hline & & & & & & 390 & 4654 & 8.4 & $>357 \mu \mathrm{mol} / \mathrm{L}$ & Female \\
\hline 61 & $\begin{array}{l}\text { Guo et al. (2017) } \\
\text { [72] }\end{array}$ & CS & Heilongjiang & Northeast & $20-59$ & 419 & 1477 & 28.4 & $>420 \mu \mathrm{mol} / \mathrm{L}$ & Male \\
\hline 62 & $\mathrm{He}$ (2017) [73] & CS & Dalian, Liaoning & Northeast & $22-91$ & 358 & 2002 & 17.9 & NS & Both \\
\hline
\end{tabular}


Table 1 Characteristics of the included studies in the systematic review and meta-analysis (Continued)

\begin{tabular}{|c|c|c|c|c|c|c|c|c|c|c|}
\hline No. & Study & $\begin{array}{l}\text { Study } \\
\text { type }\end{array}$ & $\begin{array}{l}\text { Provinces (cities)/ } \\
\text { municipalities/ } \\
\text { autonomous regions }\end{array}$ & Region & $\begin{array}{l}\text { Age } \\
\text { (years) }^{c}\end{array}$ & Case & $\begin{array}{l}\text { Sample } \\
\text { size }\end{array}$ & $\begin{array}{l}\text { Prevalence } \\
\text { (\%) }\end{array}$ & Diagnostic cut-offs & Gender \\
\hline & & & & & & 252 & 1044 & 24.1 & $>420 \mu \mathrm{mol} / \mathrm{L}$ & Male \\
\hline & & & & & & 106 & 958 & 11.1 & $\begin{array}{l}\text { premenopausal }>350 \mu \mathrm{mol} / \\
\mathrm{L} \\
\text { postmenopausal }>420 \mu \mathrm{mol} /\end{array}$ & Female \\
\hline 63 & $\begin{array}{l}\text { Li et al. (2017) } \\
\text { [74] }\end{array}$ & $\mathrm{CC}$ & Urumqi, Xinjiang & Northwest & $18-78$ & 221 & 1644 & 23.8 & NS & Both \\
\hline 64 & $\begin{array}{l}\text { Li, Zhou \& Pan } \\
\text { (2017) [75] }\end{array}$ & CS & Guangdong & $\begin{array}{l}\text { South } \\
\text { Central }\end{array}$ & $22-90$ & 314 & 3071 & 10.2 & NS & Both \\
\hline \multirow[t]{3}{*}{65} & \multirow{3}{*}{$\begin{array}{l}\text { Lin et al. (2017) } \\
\text { [76] }\end{array}$} & \multirow[t]{3}{*}{ CS } & \multirow[t]{3}{*}{ Yunnan } & \multirow{3}{*}{$\begin{array}{l}\text { South } \\
\text { Central }\end{array}$} & \multirow[t]{3}{*}{$18-84$} & 196 & 1682 & 11.7 & NS & Both \\
\hline & & & & & & 139 & 923 & 15.1 & $\geq 417 \mu \mathrm{mol} / \mathrm{L}$ & Male \\
\hline & & & & & & 57 & 759 & 7.5 & $\geq 357 \mu \mathrm{mol} / \mathrm{L}$ & Female \\
\hline \multirow[t]{3}{*}{66} & \multirow{3}{*}{$\begin{array}{l}\text { Liu et al. (2017a) } \\
\text { [77] }\end{array}$} & \multirow[t]{3}{*}{ CS } & \multirow[t]{3}{*}{ Shanghai } & \multirow[t]{3}{*}{ East } & \multirow[t]{3}{*}{$\geq 18$} & 148 & 908 & 16.3 & NS & Both \\
\hline & & & & & & 48 & 308 & 15.6 & $>420 \mu \mathrm{mol} / \mathrm{L}$ & Male \\
\hline & & & & & & 100 & 600 & 16.7 & $>360 \mu \mathrm{mol} / \mathrm{L}$ & Female \\
\hline \multirow[t]{3}{*}{67} & \multirow{3}{*}{$\begin{array}{l}\text { Liu et al. (2017b) } \\
\text { [78] }\end{array}$} & \multirow[t]{3}{*}{ CS } & \multirow[t]{3}{*}{ Shanghai } & \multirow[t]{3}{*}{ East } & \multirow[t]{3}{*}{$20-80$} & 1444 & 9294 & 15.5 & NS & Both \\
\hline & & & & & & 639 & 3393 & 18.8 & $>420 \mu \mathrm{mol} / \mathrm{L}$ & Male \\
\hline & & & & & & 805 & 5901 & 13.6 & $>357 \mu \mathrm{mol} / \mathrm{L}$ & Female \\
\hline \multirow[t]{3}{*}{68} & \multirow{3}{*}{$\begin{array}{l}\text { Liu et al. (2017c) } \\
\text { [79] }\end{array}$} & \multirow[t]{3}{*}{ CS } & \multirow[t]{3}{*}{ Hunan } & \multirow{3}{*}{$\begin{array}{l}\text { South } \\
\text { Central }\end{array}$} & \multirow[t]{3}{*}{$20-80$} & 1435 & 5356 & 26.8 & NS & Both \\
\hline & & & & & & 1234 & 3489 & 35.4 & NS & Male \\
\hline & & & & & & 201 & 1867 & 10.8 & NS & Female \\
\hline 69 & Liu, Yan \& Li & CS & Hebei & North & $\geq 18$ & 698 & 6045 & 11.5 & NS & Both \\
\hline & & & & & & 488 & 3344 & 14.6 & $>416 \mu \mathrm{mol} / \mathrm{L}$ & Male \\
\hline & & & & & & 210 & 2701 & 7.8 & $>357 \mu \mathrm{mol} / \mathrm{L}$ & Female \\
\hline 70 & $\begin{array}{l}\text { Liu \& Yang } \\
\text { (2017) [81] }\end{array}$ & $\mathrm{CC}$ & Beijing & North & $21-67$ & 204 & 1799 & 11.3 & NS & Both \\
\hline 71 & Min (2017) & CS & Shenyang, Liaoning & Northeast & & 74 & 282 & 26.2 & NS & Both \\
\hline 72 & Pan \& Jiang & CS & Fuzhou, Fujian & East & 75 & 210 & 744 & 28.2 & NS & Both \\
\hline & & & & & & 196 & 618 & 31.7 & $>420 \mu \mathrm{mol} / \mathrm{L}$ & Male \\
\hline & & & & & & 14 & 126 & 11.1 & $>420 \mu \mathrm{mol} / \mathrm{L}$ & Female \\
\hline 73 & Wang \& Bai & CS & Ningxia & Northwest & $22-60$ & 121 & 1012 & 12.0 & NS & Both \\
\hline & & & & & & 99 & 757 & 13.1 & $>420 \mu \mathrm{mol} / \mathrm{L}$ & Male \\
\hline & & & & & & 22 & 255 & 8.6 & $>357 \mu \mathrm{mol} / \mathrm{L}$ & Female \\
\hline 74 & Wang \& Bao & CS & Shanghai & East & $60-93$ & 454 & 2426 & 18.7 & NS & Both \\
\hline & & & & & & 220 & 1076 & 20.5 & $>420 \mu \mathrm{mol} / \mathrm{L}$ & Male \\
\hline & & & & & & 234 & 1350 & 17.3 & $>360 \mu \mathrm{mol} / \mathrm{L}$ & Female \\
\hline 75 & Xie et al. (2017) & CS & Guangdong & South & $35-75$ & 279 & 2587 & 10.8 & NS & Both \\
\hline & & & & & & 175 & 1410 & 12.4 & $>417 \mu \mathrm{mol} / \mathrm{L}$ & Male \\
\hline & & & & & & 104 & 1177 & 8.8 & $>357 \mu \mathrm{mol} / \mathrm{L}$ & Female \\
\hline 76 & Yu \& Jie (2017) & CS & Shandong & East & $21-76$ & 1191 & 10,743 & 11.1 & NS & Both \\
\hline & & & & & & 1116 & 6426 & 10.4 & $\geq 430 \mu \mathrm{mol} / \mathrm{L}$ & Male \\
\hline & & & & & & 75 & 4317 & 0.7 & $\geq 375 \mu \mathrm{mol} / \mathrm{L}$ & Female \\
\hline 77 & $\begin{array}{l}\text { Zhang (2017a) } \\
\text { [87] }\end{array}$ & CS & Liaoning & Northeast & $21-50$ & 121 & 500 & 24.2 & NS & Both \\
\hline 78 & $\begin{array}{l}\text { Zhang (2017b) } \\
\text { [88] }\end{array}$ & CS & Anhui & East & $25-87$ & 19 & 230 & 8.3 & $>420 \mu \mathrm{mol} / \mathrm{L}$ & Both \\
\hline
\end{tabular}


Table 1 Characteristics of the included studies in the systematic review and meta-analysis (Continued)

\begin{tabular}{|c|c|c|c|c|c|c|c|c|c|c|}
\hline No. & Study & $\begin{array}{l}\text { Study } \\
\text { type }\end{array}$ & $\begin{array}{l}\text { Provinces (cities)/ } \\
\text { municipalities/ } \\
\text { autonomous regions }\end{array}$ & Region & $\begin{array}{l}\text { Age } \\
\text { (years) }^{c}\end{array}$ & Case & $\begin{array}{l}\text { Sample } \\
\text { size }\end{array}$ & $\begin{array}{l}\text { Prevalence } \\
(\%)\end{array}$ & Diagnostic cut-offs & Gender \\
\hline \multirow[t]{3}{*}{79} & \multirow{3}{*}{$\begin{array}{l}\text { Zhang, Chen \& } \\
\text { Liu (2017) [89] }\end{array}$} & \multirow[t]{3}{*}{ CS } & \multirow[t]{3}{*}{ Zhuhai, Guangdong } & \multirow{3}{*}{$\begin{array}{l}\text { South } \\
\text { Central }\end{array}$} & \multirow[t]{3}{*}{$18-75$} & 590 & 1834 & & NS & Both \\
\hline & & & & & & 290 & 679 & 42.7 & NS & Male \\
\hline & & & & & & 300 & 1155 & 26.0 & NS & Female \\
\hline 80 & $\begin{array}{l}\text { Zheng (2017) } \\
\text { [90] }\end{array}$ & CS & China $^{a}$ & $N A^{a}$ & $24 \pm 6$ & 432 & 1721 & 25.1 & $>420 \mu \mathrm{mol} / \mathrm{L}$ & Male \\
\hline \multirow[t]{3}{*}{81} & \multirow{3}{*}{$\begin{array}{l}\text { Chen et al. } \\
\text { (2018a) [91] }\end{array}$} & \multirow[t]{3}{*}{ CS } & \multirow{3}{*}{$\begin{array}{l}\text { Liaoning, Heilonjiang, } \\
\text { Shandong, Henan, } \\
\text { Hubei, Hunan, Jiangsu, } \\
\text { Guizhou, Guangxi }\end{array}$} & \multirow[t]{3}{*}{$N A^{b}$} & \multirow[t]{3}{*}{$49 \pm 17$} & 1435 & 8785 & 16.3 & NS & Both \\
\hline & & & & & & 886 & 4110 & 21.6 & $\geq 420 \mu \mathrm{mol} / \mathrm{L}$ & Male \\
\hline & & & & & & 549 & 4675 & 11.7 & $\geq 360 \mu \mathrm{mol} / \mathrm{L}$ & Female \\
\hline 82 & $\begin{array}{l}\text { Chen et al. } \\
\text { (2018b) [92] }\end{array}$ & CS & Guangxi & $\begin{array}{l}\text { South } \\
\text { Central }\end{array}$ & $>60$ & 161 & 817 & 19.7 & $>420 \mu \mathrm{mol} / \mathrm{L}$ & Both \\
\hline 83 & $\begin{array}{l}\text { Chen et al. } \\
\text { (2018c) [93] }\end{array}$ & CS & Guangdong & $\begin{array}{l}\text { South } \\
\text { Central }\end{array}$ & $\geq 18$ & 328 & 981 & 33.4 & $>420 \mu \mathrm{mol} / \mathrm{L}$ & Male \\
\hline \multirow[t]{3}{*}{84} & \multirow{3}{*}{$\begin{array}{l}\text { Chen et al. } \\
\text { (2018d) [94] }\end{array}$} & \multirow[t]{3}{*}{ CS } & \multirow[t]{3}{*}{ Guangxi } & \multirow{3}{*}{$\begin{array}{l}\text { South } \\
\text { Central }\end{array}$} & \multirow[t]{3}{*}{$65-96$} & 241 & 1223 & 19.7 & NS & Both \\
\hline & & & & & & 163 & 629 & 25.9 & $\geq 420 \mu \mathrm{mol} / \mathrm{L}$ & Male \\
\hline & & & & & & 78 & 594 & 13.1 & $\geq 360 \mu \mathrm{mol} / \mathrm{L}$ & Female \\
\hline 85 & $\begin{array}{l}\text { Fan, Mao \& } \\
\text { Chen (2018) [95] }\end{array}$ & CS & Ningbo, Zhejiang & East & $\geq 45$ & 750 & 3395 & 22.1 & NS & Both \\
\hline \multirow[t]{3}{*}{86} & \multirow[t]{3}{*}{$\mathrm{He}(2018)$ [96] } & \multirow[t]{3}{*}{ CS } & \multirow[t]{3}{*}{ Henan } & \multirow{3}{*}{$\begin{array}{l}\text { South } \\
\text { Central }\end{array}$} & \multirow[t]{3}{*}{$25-89$} & 410 & 2193 & 18.7 & NS & Both \\
\hline & & & & & & 305 & 1156 & 26.4 & $>420 \mu \mathrm{mol} / \mathrm{L}$ & Male \\
\hline & & & & & & 105 & 1037 & 10.1 & $>350 \mu \mathrm{mol} / \mathrm{L}$ & Female \\
\hline 87 & Hu et al. (2018) & CS & Guangxi & South & $20-70$ & 1035 & 6241 & 16.6 & NS & Both \\
\hline & & & & & & 755 & 3271 & 23.1 & $>420 \mu \mathrm{mol} / \mathrm{L}$ & Male \\
\hline & & & & & & 280 & 2970 & 9.4 & $>360 \mu \mathrm{mol} / \mathrm{L}$ & Female \\
\hline 88 & Huang \& Huang & CS & Guangzhou, & South & $51-82$ & 55 & 338 & 16.3 & NS & Both \\
\hline & & & Guang & Central & & 49 & 289 & 17.0 & NS & Male \\
\hline & & & & & & 6 & 49 & 12.2 & NS & Female \\
\hline 89 & $\begin{array}{l}\text { Huang et al. } \\
\text { (2018) [99] }\end{array}$ & CS & Guizhou & Southwest & $18-75$ & $\begin{array}{l}26 \\
341\end{array}$ & 143,687 & 18.3 & NS & Both \\
\hline & & & & & & $\begin{array}{l}15 \\
387\end{array}$ & 75,364 & 20.4 & $\geq 417 \mu \mathrm{mol} / \mathrm{L}$ & Male \\
\hline & & & & & & $\begin{array}{l}20 \\
954\end{array}$ & 68,323 & 16.0 & $\geq 357 \mu \mathrm{mol} / \mathrm{L}$ & Female \\
\hline 90 & Li, Wang \& Xu & CS & Beijing & North & $18-80$ & 255 & 1700 & 15.0 & NS & Both \\
\hline & & & & & & 116 & 620 & 18.7 & NS & Male \\
\hline & & & & & & 139 & 1080 & 12.9 & NS & Female \\
\hline 91 & Lin et al. (2018a) & CS & Fujian & East & $18-63$ & 666 & 2666 & 25.0 & NS & Both \\
\hline & & & & & & 411 & 1251 & 43.9 & $>417 \mu \mathrm{mol} / \mathrm{L}$ & Male \\
\hline & & & & & & 255 & 1415 & 18.0 & $>357 \mu \mathrm{mol} / \mathrm{L}$ & Female \\
\hline 92 & Lin et al. (2018b) & $\mathrm{CS}$ & Guangzhou, & South & $\geq 18$ & 1642 & 5603 & 29.3 & NS & Both \\
\hline & & & Gl & Central & & 1590 & 5281 & 30.1 & $>420 \mu \mathrm{mol} / \mathrm{L}$ & Male \\
\hline & & & & & & 53 & 322 & 16.5 & $>350 \mu \mathrm{mol} / \mathrm{L}$ & Female \\
\hline 93 & Lu (2018a) [103] & CS & Zhejiang & East & 55 & 147 & 1200 & 12.3 & NS & Both \\
\hline & & & & & & 93 & 597 & 15.6 & $>420 \mu \mathrm{mol} / \mathrm{L}$ & Male \\
\hline & & & & & & 54 & 603 & 9.0 & $>350 \mu \mathrm{mol} / \mathrm{L}$ & Female \\
\hline 94 & Lu (2018b) [104] & $\mathrm{CH}$ & Inner Mongolia & North & $\geq 35$ & 383 & 2554 & 15.0 & NS & Both \\
\hline
\end{tabular}


Table 1 Characteristics of the included studies in the systematic review and meta-analysis (Continued)

\begin{tabular}{|c|c|c|c|c|c|c|c|c|c|c|}
\hline No. & Study & $\begin{array}{l}\text { Study } \\
\text { type }\end{array}$ & $\begin{array}{l}\text { Provinces (cities)/ } \\
\text { municipalities/ } \\
\text { autonomous regions }\end{array}$ & Region & $\begin{array}{l}\text { Age } \\
\text { (years) }^{c}\end{array}$ & Case & $\begin{array}{l}\text { Sample } \\
\text { size }\end{array}$ & $\begin{array}{l}\text { Prevalence } \\
(\%)\end{array}$ & Diagnostic cut-offs & Gender \\
\hline & & & & & & 331 & 1632 & 20.3 & $>420 \mu \mathrm{mol} / \mathrm{L}$ & Male \\
\hline & & & & & & 52 & 922 & 5.6 & $>360 \mu \mathrm{mol} / \mathrm{L}$ & Female \\
\hline & & & & & & 477 & 2554 & 18.7 & NS & Both \\
\hline & & & & & & 413 & 1632 & 25.3 & $>420 \mu \mathrm{mol} / \mathrm{L}$ & Male \\
\hline & & & & & & 64 & 922 & 6.9 & $>360 \mu \mathrm{mol} / \mathrm{L}$ & Female \\
\hline & & & & & & 511 & 2554 & 20.0 & NS & Both \\
\hline & & & & & & 446 & 1632 & 27.3 & $>420 \mu \mathrm{mol} / \mathrm{L}$ & Male \\
\hline & & & & & & 65 & 922 & 7.6 & $>360 \mu \mathrm{mol} / \mathrm{L}$ & Female \\
\hline & & & & & & 530 & 2554 & 20.8 & NS & Both \\
\hline & & & & & & 465 & 1632 & 28.5 & $>420 \mu \mathrm{mol} / \mathrm{L}$ & Male \\
\hline & & & & & & 65 & 922 & 8.0 & $>360 \mu \mathrm{mol} / \mathrm{L}$ & Female \\
\hline \multirow[t]{3}{*}{95} & \multirow{3}{*}{$\begin{array}{l}\text { Su et al. (2018) } \\
{[105]}\end{array}$} & \multirow[t]{3}{*}{ CS } & \multirow[t]{3}{*}{ Zhejiang } & \multirow[t]{3}{*}{ East } & \multirow{3}{*}{$\begin{array}{l}\text { range } \geq \\
18\end{array}$} & 694 & 3905 & 17.8 & NS & Both \\
\hline & & & & & & 364 & 1797 & 20.3 & NS & Male \\
\hline & & & & & & 330 & 2108 & 15.7 & NS & Female \\
\hline \multirow[t]{3}{*}{96} & \multirow{3}{*}{$\begin{array}{l}\text { Tuo et al. (2018) } \\
\text { [106] }\end{array}$} & \multirow[t]{3}{*}{ CS } & \multirow[t]{3}{*}{ Gansu } & \multirow[t]{3}{*}{ Northwest } & \multirow[t]{3}{*}{$20-80$} & 768 & 4263 & 18.0 & NS & Both \\
\hline & & & & & & 432 & 1783 & 24.2 & $\geq 420 \mu \mathrm{mol} / \mathrm{L}$ & Male \\
\hline & & & & & & 336 & 2480 & 13.6 & $\geq 350 \mu \mathrm{mol} / \mathrm{L}$ & Female \\
\hline \multirow[t]{3}{*}{97} & \multirow{3}{*}{$\begin{array}{l}\text { Wang et al. } \\
\text { (2018a) [107] }\end{array}$} & \multirow[t]{3}{*}{ CS } & \multirow{3}{*}{$\begin{array}{l}\text { Beijing; Xi'an, Shaanxi; } \\
\text { Harbin, Heilongjiang; } \\
\text { Chengdu, Sichuan; } \\
\text { Chongqing; Changsha, } \\
\text { Hunan; Shanghai }\end{array}$} & \multirow[t]{3}{*}{$N A^{b}$} & \multirow[t]{3}{*}{$\geq 60$} & 754 & 5351 & 14.1 & NS & Both \\
\hline & & & & & & 304 & 2304 & 13.2 & $\geq 420 \mu \mathrm{mol} / \mathrm{L}$ & Male \\
\hline & & & & & & 450 & 3047 & 14.8 & $\geq 360 \mu \mathrm{mol} / \mathrm{L}$ & Female \\
\hline \multirow[t]{3}{*}{98} & \multirow{3}{*}{$\begin{array}{l}\text { Wang et al. } \\
(2018 b) \text { [108] }\end{array}$} & \multirow[t]{3}{*}{ CS } & \multirow{3}{*}{$\begin{array}{l}\text { Liaoning; Heilongjiang; } \\
\text { Jiangsu; Shandong; } \\
\text { Henan; Hubei; Hunan; } \\
\text { Guangxi }\end{array}$} & \multirow[t]{3}{*}{$N A^{b}$} & $\geq 18$ & 555 & 4111 & 13.5 & NS & Both \\
\hline & & & & & & 361 & 1871 & 19.3 & $>418 \mu \mathrm{mol} / \mathrm{L}$ & Male \\
\hline & & & & & & 194 & 2240 & 8.7 & $>357 \mu \mathrm{mol} / \mathrm{L}$ & Female \\
\hline 99 & $\begin{array}{l}\text { Wang \& Ma } \\
\text { (2018) [109] }\end{array}$ & CS & Liaoning & Northeast & $22-65$ & 432 & 1481 & 29.2 & $>420 \mu \mathrm{mol} / \mathrm{L}$ & Male \\
\hline 100 & $\begin{array}{l}\text { Yang et al. } \\
\text { (2018) [110] }\end{array}$ & CS & China $^{a}$ & $N A^{a}$ & $\geq 18$ & 3855 & 24,095 & 16.0 & NS & Both \\
\hline 101 & $\begin{array}{l}\text { Yu et al. (2018) } \\
\text { [111] }\end{array}$ & CS & Xinjiang & Northwest & $30-81$ & 2648 & 14,426 & 18.4 & NS & Both \\
\hline 102 & Zhang et al. & CS & Ningxia & Northwest & $\geq 18$ & 3880 & 19,356 & 20.0 & NS & Both \\
\hline & & & & & & 3180 & 12,115 & 26.2 & $>420 \mu \mathrm{mol} / \mathrm{L}$ & Male \\
\hline & & & & & & 700 & 7241 & 9.7 & $>350 \mu \mathrm{mol} / \mathrm{L}$ & Female \\
\hline 103 & Zhou et al. & CS & Ningxia & Northwest & $\geq 35$ & 279 & 1743 & 16.0 & NS & Both \\
\hline & & & & & & 193 & 1044 & 18.5 & NS & Male \\
\hline & & & & & & 86 & 699 & 12.3 & NS & Female \\
\hline 104 & Hu, Zhao \& & CS & Tibet & Northwest & $20-49$ & 170 & 1669 & 10.2 & NS & Both \\
\hline & $\begin{array}{l}\text { Snang }(2019) \\
\text { [114] }\end{array}$ & & & & & 114 & 952 & 12.0 & NS & Male \\
\hline & & & & & & 56 & 717 & 7.8 & NS & Female \\
\hline 105 & $\begin{array}{l}\text { Tian et al. (2019) } \\
\text { [115] }\end{array}$ & CS & Beijing & North & 18-97 & $\begin{array}{l}10 \\
795\end{array}$ & 52,673 & 20.5 & NS & Both \\
\hline & & & & & & 8524 & 27,419 & 31.1 & NS & Male \\
\hline & & & & & & 2271 & 25,254 & 9.0 & NS & Female \\
\hline 106 & Wang & CC & China $^{a}$ & $N A^{a}$ & $\geq 18$ & 2977 & 22,983 & 13.0 & NS & Both \\
\hline & & & & & & 1999 & 10,787 & 18.5 & NS & Male \\
\hline
\end{tabular}


Table 1 Characteristics of the included studies in the systematic review and meta-analysis (Continued)

\begin{tabular}{|c|c|c|c|c|c|c|c|c|c|c|}
\hline No. & Study & $\begin{array}{l}\text { Study } \\
\text { type }\end{array}$ & $\begin{array}{l}\text { Provinces (cities)/ } \\
\text { municipalities/ } \\
\text { autonomous regions }\end{array}$ & Region & $\begin{array}{l}\text { Age } \\
\text { (years) }^{c}\end{array}$ & Case & $\begin{array}{l}\text { Sample } \\
\text { size }\end{array}$ & $\begin{array}{l}\text { Prevalence } \\
\text { (\%) }\end{array}$ & Diagnostic cut-offs & Gender \\
\hline & & & & & & 978 & 12,796 & 7.6 & NS & Female \\
\hline 107 & $\begin{array}{l}\text { Yang (2019) } \\
{[116]}\end{array}$ & $\mathrm{CH}$ & Guilin, Guangxi & $\begin{array}{l}\text { South } \\
\text { Central }\end{array}$ & $20-68$ & 160 & 1545 & 10.4 & NS & Both \\
\hline 108 & $\begin{array}{l}\text { Yu et al. (2019) } \\
\text { [117] }\end{array}$ & CS & Shenyang, Liaoning & Northeast & $\geq 18$ & 7705 & 14,323 & 53.7 & NS & Both \\
\hline
\end{tabular}

CS Cross-sectional, CC Case control, CH Cohort study, NA Not applicable, NS Not stated

${ }^{a}$ No specific provinces were reported

${ }^{\mathrm{b}}$ More than one region was involved

${ }^{\mathrm{c}}$ Mean used unless range reported

females (22.7\% (95\% CI: $20.2-25.4 \%)$ vs. $11.0 \%$ (95\% CI: 9.6-12.6\%) $(P<0.001)$ (Table 2$)$. For the study types, there was no difference in prevalence $(P=0.062)$ and the range of prevalence of hyperuricemia was from 11.9 to $18.1 \%$.

Figure 3 shows the prevalence of hyperuricemia in mainland China by different provinces, municipalities and autonomous regions. Shanghai, Jiangxi, Jilin, Liaoning, Fujian, Guangdong and Guangxi reported a high prevalence of hyperuricemia $\geq 20 \%$, while Hubei, Shandong and Shanxi had a low prevalence of hyperuricemia $<10 \%$. The remaining provinces, municipalities and autonomous regions had a moderate prevalence of hyperuricemia (10-19\%). For males, five provinces (i.e. Anhui, Guangdong, Guangxi, Jilin, and Fujian) reported a very high prevalence of hyperuricemia $\geq 30 \%$ and the remaining provinces, municipalities and autonomous regions reported a moderate-to-high prevalence of hyperuricemia $\geq 10-29 \%$. For females, majority of the provinces, municipalities and autonomous regions reported a low-to-moderate prevalence of hyperuricemia (0-19\%), while Guizhou was the only province with high prevalence of hyperuricemia $(\geq 20 \%)$.

In the general population, there was a downward trend in the prevalence of hyperuricemia from 1995 to 1999 (22.1\%) to 2015-2019 (18.6\%). Similar downwards trends in the prevalence of hyperuricemia for males and females were also observed.

\section{Analysis of heterogeneity and publication bias}

There was a significant heterogeneity in the included studies $\left(\mathrm{I}^{2}=99.735 \%, P<0.001\right)$. However, no indications of publication bias were observed as indicated by a symmetrical funnel plot (Fig. 4) and Begg and

Table 2 Prevalence of hyperuricemia by subgroups in mainland China

\begin{tabular}{|c|c|c|c|c|c|}
\hline Subgroups & No. of studies & Pooled & $95 \% \mathrm{Cl}$ & $I^{2}(\%)$ & $P$-value \\
\hline \multicolumn{6}{|l|}{ Region } \\
\hline East & 23 & 0.173 & $0.139-0.213$ & 99.844 & $<0.001$ \\
\hline North & 16 & 0.174 & $0.134-0.222$ & 99.241 & $<0.001$ \\
\hline Northeast & 6 & 0.246 & $0.163-0.353$ & 99.873 & $<0.001$ \\
\hline Northwest & 18 & 0.155 & $0.121-0.197$ & 97.447 & $<0.001$ \\
\hline South Central & 26 & 0.207 & $0.170-0.249$ & 99.373 & $<0.001$ \\
\hline Southwest & 6 & 0.158 & $0.102-0.236$ & 99.779 & $<0.001$ \\
\hline Overall & 95 & 0.181 & $0.163-0.201$ & 99.734 & 0.281 \\
\hline \multicolumn{6}{|l|}{ Sex } \\
\hline Females & 83 & 0.110 & $0.096-0.126$ & 99.678 & $<0.001$ \\
\hline Males & 89 & 0.227 & $0.202-0.254$ & 99.447 & $<0.001$ \\
\hline Overall & 172 & 0.163 & $0.149-0.178$ & 99.613 & $<0.001$ \\
\hline \multicolumn{6}{|l|}{ Study type } \\
\hline Cross-sectional & 94 & 0.181 & $0.164-0.200$ & 99.761 & $<0.001$ \\
\hline Cohort & 9 & 0.119 & $0.082-0.169$ & 95.073 & $<0.001$ \\
\hline Case control & 4 & 0.149 & $0.088-0.240$ & 94.186 & $<0.001$ \\
\hline Overall & 107 & 0.174 & $0.158-0.191$ & 99.735 & 0.062 \\
\hline
\end{tabular}




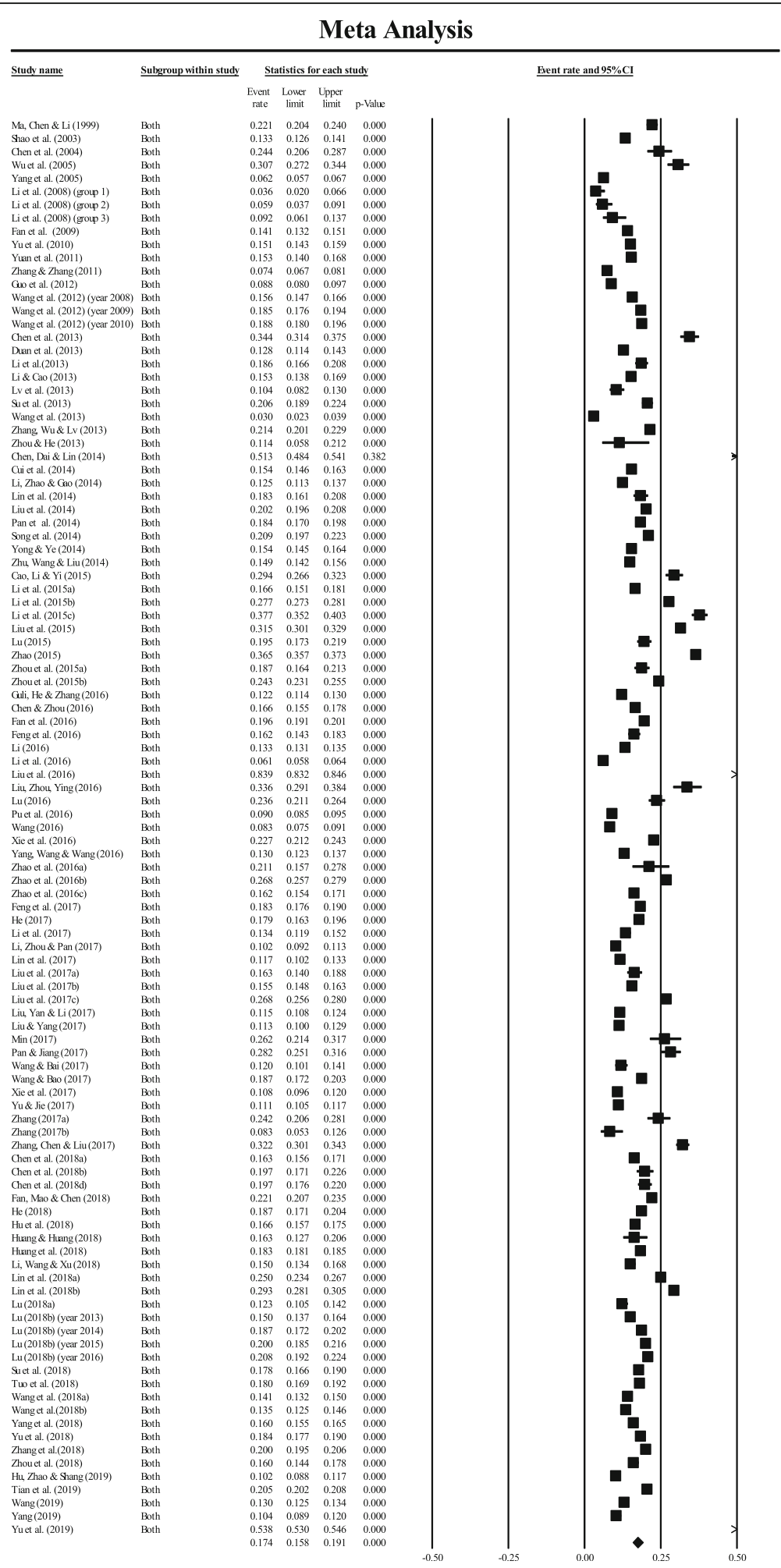

Fig. 2 Forest plot of the pooled prevalence and $95 \% \mathrm{Cl}$ of hyperuricemia among the general population in mainland China 


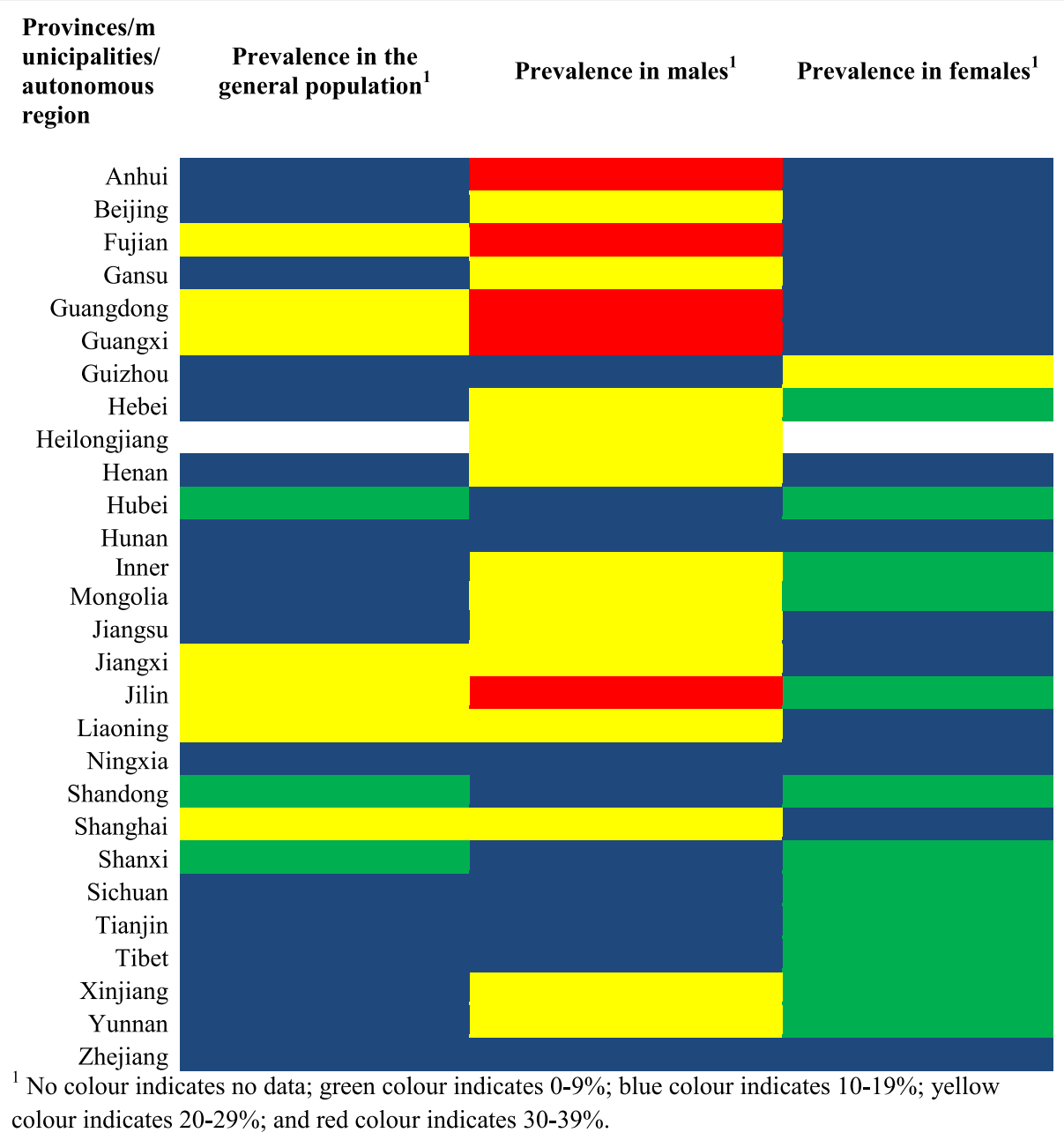

Fig. 3 Prevalence of hyperuricemia in mainland China according to different provinces, municipalities and autonomous regions

Mazumdar rank correlation $(P=0.392)$. The overall results remained unchanged as well after we performed a trim and fill method. Similarly, no publication bias was also reported for the subgroups analysis (Begg and Mazumdar rank correlation with a $P$-value $>0.05$ ) and all funnel plots were symmetrical.

\section{Discussion}

We performed a comprehensive meta-analysis of 108 observational studies over two decades and covered 27 provinces, autonomous regions and municipalities in the mainland China. In our meta-analysis, the prevalence of hyperuricemia in the general population of mainland China was $17.4 \%$ (22.7\% in males and $11.0 \%$ in females), which was within the range of reported global prevalence (ranging from 1 to $85 \%$ ) [8].

Our pooled prevalence was higher than a meta-analysis reported by Liu et al. i.e. 13.3\% (19.4\% in males and 7.9\% in females) [11]. Our prevalence was similar to some developing countries in Asia. In Thailand, the overall prevalence of hyperuricemia was $10.6 \%$ in the general population with 18.4 and $7.8 \%$ in males and females, respectively [124]. In Turkey, the overall prevalence of hyperuricemia was $12.1 \%$ and males had a higher prevalence than females (i.e. $19.0 \%$ vs. $5.8 \%$ ) [125].

However, our results were lower than that reported in developed countries [122, 126]. In the United States, the prevalence of hyperuricemia was 21.2 and $21.6 \%$ in males and females, respectively [126]. In Japan, the prevalence of hyperuricemia in the general population was $25.8 \%$ ( 34.5 and $11.6 \%$ in males and females, respectively) [122]. The higher prevalence reported in developed countries was most likely due to rapid aging and urbanisation [126]. In addition, the prevalence of noncommunicable disease and obesity has also increased in these developed countries [122, 126], which might have contributed to the higher prevalence of hyperuricemia. Therefore, we strongly recommend that the Chinese health authorities should introduce more effective public health policies measures including prevention of obesity 


\section{Funnel Plot of Standard Error by Logit event rate}

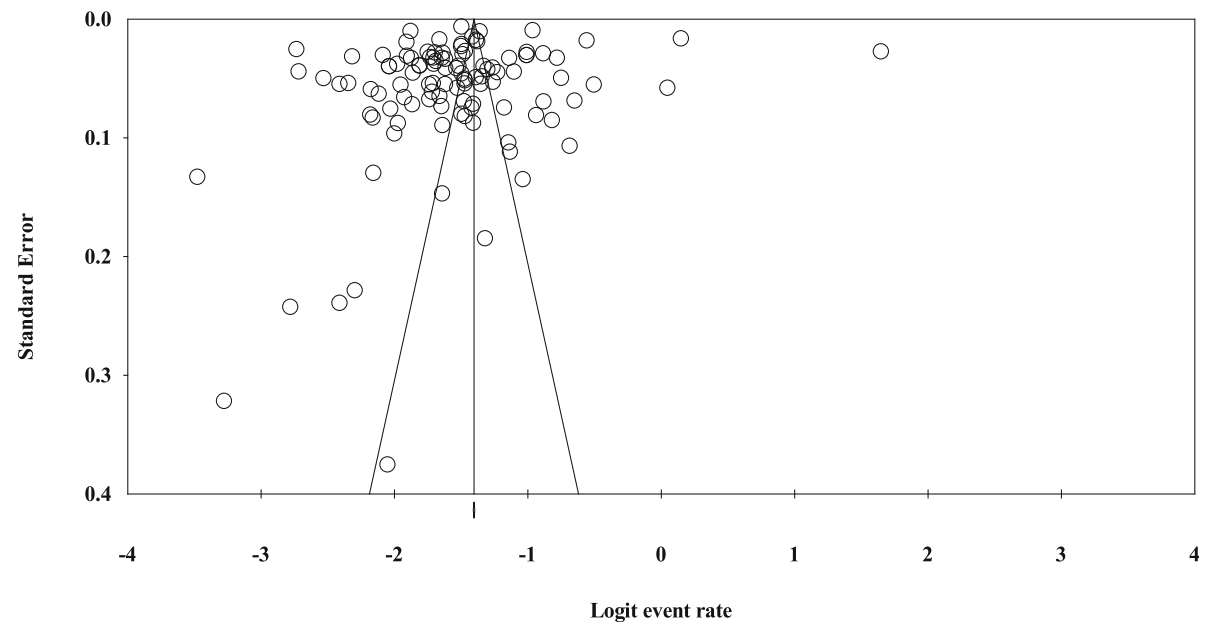

Fig. 4 Funnel plot for the meta-analysis of the prevalence of hyperuricemia in mainland China

programme and promotion of health lifestyles to reduce the prevalence of hyperuricemia in Chinese population.

Since China is a vast country characterised by distinct regions, the prevalence of hyperuricemia varies largely in different provinces and regions. Our results reported that the prevalence of hyperuricemia ranged from 15.8 to $24.6 \%$, with the highest prevalence in the Northeast region. We postulated that the large variability in the prevalence might be caused by the difference in the economic development and sedentary lifestyle adopted in these regions and provinces. For example, those living in Guangxi, Guangdong, Fujian and Jiangxi, people would consume more meat, alcohol and seafood. These foods are rich in purine which can cause an increase in the production of uric acid in the body [127]. Shanghai is one of the most economically developed areas in China. Rapid economic growth has led to unhealthy lifestyles and dietary patterns in the Shanghai population. In addition, an increased inactivity at work has also contributed to a higher prevalence of hyperuricemia [128]. In Jilin and Liaoning, we also reported a high prevalence of hyperruricemia (20-29\%), which could be due to the high consumption of alcohol intake, particularly beer and liquor [129]. However, the specific reasons why these regions had a high prevalence require further research. In addition, with these results, the management of hyperuricemia (including routine health check-ups and serum uric acid screening tests) in these regions can be better implemented and improved by the health authorities. Nutrition education and lifestyle interventions can also be developed and specifically targeted to the high risk regions with proper healthcare resources by the health authorities. This is because if hyperuricemia is not well managed and prevented especially in regions with high prevalence, it can induce several medical complication including chronic failure and gout, which increases the cost of medical care [2].

In addition, we reported that males had a significantly higher prevalence of hyperuricemia than females $(22.7 \%$ vs. $11.0 \%$ ). Such a difference might be due to the sex hormones [130]. Serum uric acid level is generally higher in males than females. This is because there is an increase renal urate clearance by estrogen in women [129]. Our findings were consistent with the results reported in several countries from Asia and the Asia Pacific region including Nepal [131], Thailand [132], Turkey [125], Saudi Arabia [133], Seychelles [134], Japan [122] and New Zealand [135].

Our study also reported an increasing prevalence of hyperuricemia over time in males and females. We speculated that factors including aging population and obesity have contributed to the increase [126]. However, we also noticed different diagnostic cut-offs were used to diagnose hyperuricemia. It will be helpful to compare these different cut-offs in the same population in order to understand their validity in diagnosing hyperuricemia.

Our meta-analysis has several strengths. Firstly, to our knowledge, our study is the most comprehensive study among the general population in mainland China. Unlike the previous two meta-analyses $[10,11]$, our sample size (>808,505 participants) and number of eligible articles ( $n=108)$ were larger; and we included analyses on differences across regions, provinces, sex and study periods. Secondly, our pooled data covered all the six regions in China. In addition, all the provinces, municipalities and autonomous regions were also included, except for Qinghai, Chongqing, Hong Kong, Macao and Hainan. Thirdly, the authors who were involved in the data extraction and interpretation were proficient in the Chinese language. However, our study also suffered from a 
few limitations. Most of the included articles were crosssectional studies. Since the definition of hyperuricemia varied according to the diagnostic cut-offs used by different studies, this factor should also be taken into consideration when interpreting these results. There was also a large heterogeneity in the quality of the articles, although no indications of publication bias were reported. We also did not make a clear distinction between urban and rural areas. Therefore, future studies with larger populations should consider investigate if health literacy, health status, sociodemographics and physical activity level play an important factor in the prevention and management of hyperuricemia, especially in adolescents, pregnant women and older adults with lower socioeconomic status [136].

\section{Conclusions}

Hyperuricemia has become an important public health problem in mainland China, particularly among males. Special attention should be paid to the residents in geographical regions with high prevalence of hyperuricemia. In addition, our study was the first comprehensive study to investigate the overall prevalence of hyperuricemia in mainland China covering the six regions. Our study also underline the importance of having more larger population-based intervention studies to tackle the increasing problem of hyperuricemia, particularly the vulnerable groups in mainland China. Future studies should investigate the association between the prevalence of hyperuricemia and its risk factors such as geographical region, economic level and sex in order to develop public health policies for tackling the issue.

\section{Abbreviations \\ CMA: Comprehensive Meta-Analysis; CVD: Cardiovascular disease; SST R: Shanghai Science and Technology Innovation Resources Center; CNKI: China National Knowledge Infrastructure; CQVIP: Chinese Scientific Journals Fulltext Database; PRISMA: Preferred Reporting Items for Systematic Reviews and Meta-Analyses; NOS: Newcastle-Ottawa Scale; Cl: Confidence intervals}

\section{Acknowledgements}

Jiayun Huang would like to thank her parents for providing continuous support in her study.

\section{Authors' contributions \\ Conceptualization: ZFM \& JH. Methodology: JH, ZFM, YZ, ZW, YL, HZ, AC \& YYL. Formal analysis: JH, ZFM, YZ, YL, HZ \&AC. Roles/Writing - original draft: $J H$ \& ZFM; Writing - review \& editing: JH, ZFM, YZ, ZW, YL, HZ, AC \& YYL. All authors read and approved the final manuscript.}

\section{Funding}

The authors would like to thank Xi'an Jiaotong-Liverpool University for providing support and funding for this hyperuricemia project (SURF code no. 76). The funder had no role in the design of this study and will not have any role during its execution, analyses, interpretation of the data, or decision to submit results.

Availability of data and materials Not applicable.
Ethics approval and consent to participate

Not applicable.

\section{Consent for publication}

Not applicable.

\section{Competing interests}

The authors declare that they have no competing interest.

\section{Author details}

'Department of Health and Environmental Sciences, Xi'an Jiaotong-Liverpool University, Suzhou 215123, Jiangsu, China. ${ }^{2}$ School of Medical Sciences, Universiti Sains Malaysia, 16150 Kota Bharu, Kelantan, Malaysia. ${ }^{3}$ Jinzhou Medical University, Jinzhou 121001, Liaoning, China. ${ }^{4}$ Department of Nutrition and Food Hygiene, School of Public Health, Soochow University, 199 Ren'ai Road, Suzhou 215123, China. ${ }^{5}$ Department of Respiratory and Critical Care Medicine, The Second People's Hospital of Wuhu, Wuhu 241000, Anhui, China. ${ }^{6}$ Clinical Medical College, Yangzhou University, Yangzhou 225009, Jiangsu, China. ${ }^{7}$ Department of Clinical Nutrition, Northern Jiangsu People's Hospital, Yangzhou 225001, Jiangsu, China. ${ }^{8}$ Department of Human Nutrition, University of Otago, Dunedin 9016, New Zealand. ${ }^{9}$ Gut Research Group, Faculty of Medicine, Universiti Kebangsaan Malaysia, 56000 Kuala Lumpur, Malaysia. ${ }^{10}$ St George and Sutherland Clinical School, University of New South Wales, Sydney, NSW 2217, Australia.

Received: 22 June 2020 Accepted: 28 October 2020

Published online: 30 November 2020

\section{References}

1. Puig JG, Martinez MA. Hyperuricemia, gout and the metabolic syndrome. Curr Opin Rheumatol. 2008;20:187-91.

2. Huang J, Ma ZF, Tian Y, Lee YY. Epidemiology and prevalence of gout in mainland China: an updated systematic review and meta-analysis. SN Compr Clin Med. 2020. https://doi.org/10.1007/s42399-020-00416-8.

3. Ichida K, Matsuo H, Takada T, et al. Decreased extra-renal urate excretion is a common cause of hyperuricemia. Nat Commun. 2012:3:764.

4. Zhou H, Ma ZF, Lu Y, et al. Elevated serum uric acid, hyperuricaemia and dietary patterns among adolescents in mainland China. J Pediatr Endocrinol Metab. 2020;33:487-93.

5. Degli Esposti L, Desideri G, Saragoni S, Buda S, Pontremoli R, Borghi C. Hyperuricemia is associated with increased hospitalization risk and healthcare costs: evidence from an administrative database in Italy. Nutr Metab Cardiovasc Dis. 2016;26:951-61.

6. Ndrepepa G. Uric acid and cardiovascular disease. Clin Chim Acta. 2018;484: 150-63.

7. Trinchieri A, Montanari E. Biochemical and dietary factors of uric acid stone formation. Urolithiasis. 2018:46:167-72.

8. Smith E, March L. Global prevalence of hyperuricemia: a systematic review of population-based epidemiological studies (abstract). Arthritis Rheumatol. 2015;67:10.

9. Li Y, Zeng P, Zhu M, Liu X, Li D. The clinical characteristics of gout attack and its complications of lower extremity deep vein thrombosis in elderly patietns during hospitalisation. Chin J Clin Healthc. 2018;21:398-401.

10. Liu B, Wang T, Zhao H, et al. The prevalence of hyperuricemia in China: a meta-analysis. BMC Public Health. 2011;11:832.

11. Liu R, Han C, Wu D, et al. Prevalence of hyperuricemia and gout in mainland China from 2000 to 2014: a systematic review and meta-analysis. Biomed Res Int. 2015;2015:762820.

12. Moher D, Liberati A, Tetzlaff J, Altman DG. Preferred reporting items for systematic reviews and meta-analyses: the PRISMA statement. Open Med. 2009;3:e123-30.

13. Higgins JP, Thompson SG, Deeks JJ, Altman DG. Measuring inconsistency in meta-analyses. BMJ. 2003;327:557-60.

14. Ma W, Chen W, Li X. Investigation and analysis of hyperuricemia in elderly population in Guangzhou. Guangzhou Med J. 1999;30:65-6.

15. Shao J, Mo B, Yu R, Li Z, Liu H, Xu Y. Epidemiological study on hyperuricemia and gout in community of Nanjing. Chin J Dis Control Prev. 2003;7:305-8.

16. Chen M, Yang M, Wang $C$, et al. Relationship study of uric acid concentration of first-degree relatives of individuals with type 2 diabetes with metabolic syndrome. J Clin Intern Med. 2004;21:806-8. 
17. Wu Z, Chen L, Zhao C, Peng C, Xiong Q. Serum uric acid level in middleaged and elderly residents from the conjoining area between city and countryside in Guangzhou and association with risk factors of other cardiovascular diseases. Chin J Clin Rehabil. 2005:9:150-2.

18. Yang $Y$, Liu $X$, Xie $H$, et al. Association between prevalence rate of hyperuricemia and risk factors of cardiovascular disease in the population of Qingdao port. Chin J Clin Rehabil. 2005;9:1-3.

19. Wang $Y$, Zhao $S$, Chen $X$, et al. Influencing factors of serum uric acid and the critical concentration of serum uric acid to prevent and treat metabolic syndrome in female inhabitants from coastal area of Shandong province. Chin J Clin Rehabil. 2006:20:147-51.

20. Li Y, Zhao D, Liu J, Li ZA, Yong Q, Wang W. The association between hyperuricemia and prevalence of carotid plaque. Zhonghua Nei Ke Za Zhi. 2008:47:906-9.

21. Lu S, Pang W, Gao S, Lu H, Peng S, Jiang Y. The nutrition survey and energy expenditure in workers on offshore oil recovery platforms. Acta Nutr Sin 2010;32:141-4

22. Yu J, Yang T, Diao W, et al. Epidemiological study on hyperuricemia and gout in Foshan areas, Guangdong province. Chin J Epidemiol. 2010;31:860-2.

23. Yuan J, Shen Y, Hu Y, Cha Y. Epidemiological investigation of chronic kidney disease in the elderly in Guiyang City. Chin J Gerontol. 2011;31:1408-10.

24. Zhang $Q$, Zhang $L$. Research on noninfectious chronic disease of teachers from colleges and universities in China. Health Med Res Pract. 2011;08:4-7.

25. Guo W, Xioa C, Shen X, Liu G, Zhang H. Prevalence of hyperuricemia and its relationship to hypertension, hyperglycemia and hyperlipidemia in community residents in Taiyuan city. Chin Gen Pract. 2012;15:3045-7.

26. Wang L, Yuan N, Li X, Zhang K, Ma B. Analysis of health examination diseases of professional population in Yinchuan city from 2008 to 2010. J Ningxia Med Univ. 2012;34:627-9.

27. Chen Y, Luo Z, Deng Z, et al. 2012 health monitoring report of residents in Binyang County, Guangxi. J Applied Prev Med. 2013;19:300-2.

28. Duan W, Zhang J, Ma Y, Cheng J. Prevalence and influencing factors of hyperuricemia among residents in Korla region of Xinjiang. Chin Gen Pract. 2013;16:916-8

29. Li L, Huang H, Liang B, Chen X, Cai H, Li X. Prevalence survey of hyperuricemia and its association with hypertriglyceridemia and hypertension in elderly people in Quanzhou of Fujian Province. Chin Geriatri. 2013:32:338-40.

30. Li H, Cao X. Analysis on the prevalence rate and influencing factors of hyperuricemia in a company in Karamay city. Med Inf. 2013;32:220-1.

31. Lv C, Mou S, Ju J, He Z, Yi Y. Hypertension characteristics and renal damage of farmers in Yantai development zone. Chin J Integr Trad West Nephrol. 2013:14:881-3.

32. Su L, Xie B, Fan $Y$, Cha H. Analysis on the prevalence of hyperuricemia and related diseases among middle-aged and elderly cadres in the South China Sea. Chin J Clin Res. 2013;26:309-12.

33. Wang J, Gu H, Lu S, Xing Y, Qin L. Epidemiological survey of prevalence of hyperuricemia and its risk factors in adult population of Chongming District, Shanghai. J Chin Physician. 2013;15:1616-8.

34. Zhang C, Wu H, Lv C. Analysis of hyperuricemia and its risk factors in physical examination group. Chin J Gerontol. 2013;33:4048-9.

35. Zhou Q, He Z. Association analysis between serum uric acid levels and cerebral infarction, as well as serum glucose, blood pressure, blood lipids and c-reactive protein. Med Philos. 2013:34:29-32.

36. Chen S, Dai H, Lin A. Relationship between hyperuricemia and cardiovascular risk factors among middle-aged and elderly persons. Chin J Public Health. 2014:30:144-8.

37. Cui S, Wang X, Tian Z, Wei H, Tian X, Yi Y. Epidemiological study on hyperuricemia and gout in community of $Y u$ County in Hebei Province. Chin J Traumatol. 2014:24:501-15.

38. Li S, Zhao Y, Gao X. Uric acid and associated factors of staff in Yunnan University. Chin J School Health. 2014;35:1199-203.

39. Lin X, Zou R, Li X, Zeng N. Prevalence survey and risk factors of hyperuricemia for rural elderly people of Licheng street in Zengcheng city. China Med Herald. 2014;11:101-4.

40. Liu J, Zhang X, Tan L, Song C, Zheng W. Investigation on the prevalence of hyperuricemia in physical examination population in Changchun and analysis of related risk factors. Chin J Clin Res. 2014;27:763-5.

41. Pan Y, Qiang D, Ding J, Shen Y. Analysis of the prevalence and influencing factors of hyperuricemia in Wujin district. Chin J Prev Control Chronic Dis. 2014;22:315-7.
42. Song W, Liu J, Chen Z, Huo Y, Lin A, Zhang Y. Hyperuricemia and gout: a prevalence survey among over-40-year-old community residents in Nanchang district. Chin Gen Pract. 2014;17:181-4.

43. Yong $X$, Ye $Y$. Analysis of uric acid test results in the blood of freshmen in a university. Chin J School Health. 2014;35:1430-1.

44. Zhu M, Wang M, Liu J. High uric acid hematic disease and renal damage epidemiological studies in Urumqi CHECK-UP CROWD. Chin J Integr Trad West Nephrol. 2014;15:125-8.

45. Cao X, Li X, Yi G. Correlation analysis of serum uric acid and blood lipid in 988 patients. Chin J Conv Med. 2015:8:851-2.

46. Li M, Liu J, Huang W, Zhang Q, et al. The relationship of hyperuricemia level with prediabetes and diabetes in Lanzhou. Chin J Diabetes. 2015a;23:11-4.

47. Li R, Li W, Wang Y, Jiang F, Chen H, Tu Q. Analysis on prevalence and influence factors of hyperuricemia among residents in Liuzhou. Chin J Health Lab Technol. 2015b;25:2807-9.

48. Li Y, Zhong G, Li Y, et al. Hyperuricemia prevalence analysis of township community residents in Dongguan. Chin Prim Health Care. 2015c;29:29-30,5

49. Lu F. The correlative study on the relative risks of hyperuricemia of the elderly in community. Chin J Ethnomed Ethnopharm. 2015;2:76-7.

50. Zhao X. Analysis of hyperuricemia prevalence and related risk factors in cnooc employees. China: Tianjin Medial University; 2015. [Thesis].

51. Zhou A, Pan Q, Li A, et al. Predictive value of obesity and metabolism indexes for hyperuricemia among rural adult Yi residents in Liangshan region. Chin J Public Health. 2015a;31:1646-50.

52. Zhou M, Yang L, Kang L, Xu J, Xie L. Prevalence and influence factors of hyperuricemia among workers in Zhengzhou railway bureau. Chin J Health Lab Technol. 2015b;25:2599-601.

53. Guli A, He M, Zhang C. The incidence and related factors of fatty liver in minority population in Gansu province. Chin J Gerontol. 2016;36:3293-4.

54. Chen $\mathrm{H}$, Xing $\mathrm{Y}$. Correlation between hyperuricemia and hypertension of male faculty in a university. Chin J School Doct. 2016;30:344-5.

55. Chen $\mathrm{N}$, Zhou J. Logistic regression analysis of risk factors of hyperuricemia in an elderly physical examination group in Shaoxing. Chin J Gerontol. 2016;36:4225-6.

56. Fan N, Zhang $\mathrm{L}$, Xia Z, et al. Association between serum uric acid and nonalcoholic fatty liver disease. Chin J Diabetes. 2016;24:678-82.

57. Feng $T$, Tian $F, W u X$, et al. Association analysis between serum uric acid and metabolic syndrome components. Chin J Diabetes. 2016;24:317-20.

58. Li Z Cardiovascular health behavior and factors associated with hyperuricemia. China: Tianjin Medical University; 2016. [Thesis].

59. Li Q, Chen H, Lu A, et al. Investigation on the incidence of hyperuricemia in Qijiang district, Chongqing. China Health Care Nutr. 2016;26:305-6.

60. Liu X, Zhou Y, Ruan X, et al. Prevalence and risk factors of chronic kidney disease among residents from Pudong new area, Shanghai. Chin Gen Pract. 2016;19:3742-50.

61. Liu Y, Zhou F, Yin X. Analysis of the rate of hyperuricemia in Zhaotong city level cadres. Chinese Comm Doct. 2016;32:139-41.

62. Lu Y. Analysis of the related factors of uric acid level and uric acid increase in elderly people. China Health Care Nutr. 2016;1:370-1.

63. Pu H, Bai $Y$, Zhao G, et al. Study on the prevalence and influencing factors of cholelithiasis in Jinchang cohort. Chin J Health Stat. 2016;33:94-6.

64. Wang Y. Relationship between high blood pressure and hyperuricemia in freshmen in a university. Chin J School Health. 2016;37:1588-9.

65. Xie Z, Jiang M, Tang J, Wei X, Wang T, Yao J. Correlation between hyperuricemia and cardiovascular risk factors in occupational population of a certain enterprise. China J Health Lab Technol. 2016:26:1980-2.

66. Yang $X$, Wang $T$, Wang $Y$. Prevalence and influencing factors of dyslipidemia among adult residents in Tianjin city: a cross-sectional study. Chin J Public Health. 2016;32:286-90.

67. Zhang C. Analysis of the prevalence of hyperuricemia and dietary factors among male freshmen in a university. In: Proceedings of the 2015 national sports health care and rehabilitation academic conference; 2016. p. 78-81.

68. Zhao X, Liu L, Wan Q, Min C, Gao J, Zhang Y. The prevalence rate of carotid atherosclerosis and related risk factors among middle-aged and elderly teachers of different ethnic groups in colleges and universities. Chin J Gerontol. 2016a;36:5142-4.

69. Zhao B, Nie Q, Qu F, Cui H, Liu J. The study on the correlation of asymptomatic hyperuricemia and blood lipid among young people. Chin Exp Diagn. 2016b;20:1486-9.

70. Zhao B, Qu F, Cui H, Yang L, Qu H, Liu X. Clinical research of relationship between blood uric acid level and biochemical indicators in faculty of Beijing university. Chin J Clin Healthc. 2016c;19:574-7. 
71. Feng W, Li H, Tao L, et al. Relationship between metabolic syndrome and hyperuricemia in physical examination population in a hospital of Beijing. Chin J Cardiovasc Med. 2017;22:438-41.

72. Guo H, Fu J, Bao C, et al. Association of fasting plasma glucose and hyperuricemia among young and middle-aged occupational men, Harbin. Modern Prev Med. 2017:44:1941-58.

73. He Y. Analysis of hyperuricemia and its risk factors in Dalian university staff. Chin J School Health. 2017:38:1594-6.

74. Li Y, He P, Zhou J, Wang S, Hailati J, Yati M. Prevalence and risk factor of non-alcoholic fatty liver disease in Xinjiang Urumai region. Chin J Clin Healthc. 2017;20:574-8

75. Li W, Zhou Y, Pan C. Study on related indexes changes of non-alcoholic fatty liver disease and metabolic syndrome. China Pract Med. 2017;12:1-3.

76. Lin J, Liu J, Zhang S, Jiang A. Analysis of the prevalence situation and related diseases of hyperuricemia in physical examination population in Puer city. Chin Comm Doct. 2017;33:114-5.

77. Liu J, Shen P, Yu Z, et al. Prevalence and risk factors of hyperuricemia in a community population of Pudong District in Shanghai. Chin J Integr Trad West Nephrol. 2017a;18:401-4.

78. Liu X, Gu J, Ruan X, et al. Prevalence and risk factors of hyperuricemia in Pudong new district of Shanghai. Chin J Prev Control Chronic Dis. 2017b;25: 165-70.

79. Liu P, Zhao Q, Chen H, Zhang G, Huang T, Xiao L. Study on body mass index and blood pressure, blood lipid, blood glucose and uric acid in Xiangtan city. China Health Care Nutr. 2017c;27:72-3.

80. Liu J, Yan W, Li Z. Study on body mass index and health status of 6045 employees in Shijiazhuang city. Chin J Prac Intern Med. 2017:37:437-9.

81. Liu Y, Yang L. Analysis of the prevalence of nonalcoholic fatty liver disease and its related diseases among faculties in a certain university in Beijing. China Med Herald. 2017;14:178-80.

82. Pan J, Jiang F. Serum uric acid level in elderly population from a city and its effect on renal function. Guide China Med. 2017:15:12-3.

83. Wang Y, Bai Y. Investigation on blood uric acid examination results of 1012 oil workers and analysis of related risk factors. Chinese Comm Doct. 2017:33:128,30.

84. Wang H, Bao X. Morbidity and risk factors of hyperuricemia in elderly people from a Shanghai community. Chin J Integr Trad West Nephrol. 2017; 18:869-72.

85. Xie $Y$, Luo R, Song $Y$, He X. Prevalence and influence factors of hyperuricemia among residents in eastern mountain area of Guangdong province. Chin J Public Health. 2017;33:317-20.

86. Yu K, Jie $X$. Investigation and analysis on the prevalence of hyperuricemia in physical examination group. China Rural Health. 2017;18:28.

87. Zhang M. Analysis of health examination results of 500 cadres in a certain army. China Health Care Nutr. 2017a;27:289-90.

88. Zhang L. Epidemiological survey on hypertension among employees of Wuhu power generation co., LTD in 2015. Chin J Rural Med Pharm. 2017b; 24:63-4.

89. Zhang L, Chen S, Liu X. Community prevalence of hyperuricemia and its comorbidities. J Inner Mongolia Med Univ. 2017;39:516-9.

90. Zheng $X$. The present status of hyperuricemia in civil aviation flying personnel. Chin J Aerosp Med. 2017;28:23-8.

91. Chen HG, Sheng LT, Wan ZZ, et al. The relationship between smoking and hyperuricemia in Chinese residents. Zhonghua Yu Fang Yi Xue Za Zhi. 2018, 52:524-9.

92. Chen $Y$, Tang Z, Fang Z, et al. Analysis of biological indicators of long-lived elderly in five long-lived areas in Guangxi. Chin J Gerontol. 2018b;38:3259-62.

93. Chen $\mathrm{S}$, Zhao $\mathrm{N}$, Li H, Guang $\mathrm{H}$, Wang $\mathrm{H}$. Influence of shift work on common risk factors of cardiovascular disease in male workers in petrochemical enterprises. China Occup Med. 2018c;45:316-20.

94. Chen H, Qin C, He Z, Chen Y, Huang H, Feng M. Study on the correlation between body mass index, uric acid and dyslipidemia on the elderly of Yao nationality in Gongcheng, Guangxi. Chin J New Clin Med. 2018d;11:338-41.

95. Fan J, Mao Y, Chen X. Analysis of risk factors affecting thyroid nodules in middle-aged and el-derly people in Ningbo. China Mod Doct. 2018;56:81-7.

96. He S. Analysis of prevalence and influential factors of fatty liver in faculty staff in a university. Chin J Health Lab Technol. 2018;28:1016-22.

97. Hu M, Liu J, Zhou C, Li X. Analysis of risk factors of hyperuricemia based on classification regression tree model. Chin Gen Pract. 2018;21:283-8.

98. Huang $\mathrm{H}$, Huang Q. Analysis of physical examination of retired army cadres and health care countermeasures. Chin J Conv Med. 2018;27:606-8.
99. Huang J, Li M, Yang Y, et al. Prevalence and influence factors of hyperuricemia among rural residents in Qiannan minority regions of Guizhou province. Chin J Public Health. 2018:34:29-33.

100. Li $P$, Wang $X, X u$ J. The prevalence of HUA and its influencing factors in community outpatients. China Health Stand Mgmt. 2018;9:11-3.

101. Lin S, Lai S, Huang Z, Yang L, Wu H. Dietary patterns and influencing factors of hyperuricemia among adult residents in Fujian province: a classification tree analysis. Chin J Public Health. 2018a;34:798-802.

102. Lin Q, Qiu C, Li Y, et al. Influencing factors on prevalence of chronic diseases in workers under high-temperature condition in a port of Guangzhou City. China Occup Med. 2018b;45:329-34.

103. Lu Z. Analysis of the prevalence and influencing factors of hyperuricemia in community residents. Chin J Rural Med Pharm. 2018a;25:52-3.

104. Lu H. Survey for four consecutive years uric acid levels and related factors in a company in the Ordos region population. Chin J Prim Med Pharm. 2018b; 25:52-7.

105. Su Y, Ni J, Zheng J, Huang X, Wu W, Xu Z. Investigation on metabolic syndrome and related chronic diseases in residents of Ouhai district. Chin J Prev Control Chronic Dis. 2018;26:597-600.

106. Tuo Y, Ren X, Li G, et al. Prevalence and impact factors of hyperuricmeia among Han adults in Gansu province. Chin J Public Health. 2018;34:808-11.

107. Wang R, Tang Z, Sun F, Diao L. Prevalence of hyperuricemia in the elderly in 7 areas of China. Chin J Epidemiol. 2018a;39:286-8.

108. Wang Y, Tian F, Wen J, Guo X, Yang X. Effect of total energy and three major nutrients intake and their changes on serum uric acid level. Sichuan Med J. 2018b;39:383-9.

109. Wang J, Ma M. Physical health among male civil aviation aircrews in China. Chin J Public Health. 2018:34:1174-7.

110. Yang Y, Zhou W, Zhang D, Zhou R, Wang Y. Correlation between hyperuricemia and chronic kidney disease in different sexes. Chin J Arterioscl. 2018;26:825-30.

111. Yu R, Wu J, Yang $X$, Chen J, Wang S. Analysis of related factors of blood lipid levels and elevated triglycerides in hyperuricemia population in healthy subjects. Med Inf. 2018;31:62-4.

112. Zhang H, Zhao X, Meng L, Dong J, Liu L. Relationship between hyperuricemia and metabolic syndrome in healthy people in Ningxia. Chin Prev Control Chronic Dis. 2018;26:660-3.

113. Zhou Y, Song Q, Li W, Li X, Wang S, Dai X. Analysis on health examination of senior technical personnel in Ningxia in 2015. J Ningxia Med Univ. 2018; 40:137-41.

114. Hu Y, Zhao C, Shang J. Disease composition analysis of sanitariums in Tibetan areas in a sanitarium from 2015 to 2017. Chin J Conv Med. 2019;28:222-4.

115. Tian Q, Wang Y, Li Z, et al. Correlation between uric acid and risk factors of cardiovascular disease in people under physical examination. China Contin Med Educ 2019:11:62-5.

116. Yang $\mathrm{H}$. Analysis of physical examination results and suggestions on health management of staff in a university in Guilin. China Health Care Nutr. 2019; 29:311.

117. Yu Z, Chen L, Xiao J, Wang L. Detection and risk factors of fatty liver in 20316 physical examinees in Jinqiu Hospital of Liaoning Province. Chin Pract Rural Doct. 2019;26:63-6.

118. Fan XH, Sun K, Wang YB, et al. Prevalence and associated risk factors of hyperuricemia in rural hypertensive patients. Natl Med J China. 2009:89: 2667-70.

119. Fu J, Huang Y, Liu J. Effects of high-intensity interval training on serum uric acid and muscle content in sea crew with hyperuricemia. Zhonghua Hang Hai Yi Xue Za Zhi. 2017;24:407-8.

120. Liu $X$, Han $Y$, Jiang J. Investigation on the current situation of hyperuricemia associated with related diseases in a university staff in Guangdong province in 2013. Chin J School Doct. 2015;29:412-3.

121. Min L. Analysis of hyperuricemia prevalence and related risk factors in idiopathic membranous nephropathy patients. China: China Medical University; 2017. [Thesis].

122. Nagahama K, Iseki K, Inoue T, Touma T, Ikemiya Y, Takishita S. Hyperuricemia and cardiovascular risk factor clustering in a screened cohort in Okinawa, Japan. Hypertens Res. 2004;27:227-33.

123. Wang D, Yi Y, Qiu L, et al. The serum uric acid is related to hypertension in Chinese population. Basic Clin Med. 2019:39:182-6.

124. Lohsoonthorn V, Dhanamun B, Williams MA. Prevalence of hyperuricemia and its relationship with metabolic syndrome in Thai adults receiving annual health exams. Arch Med Res. 2006:37:883-9. 
125. Sari I, Akar S, Pakoz B, Sisman AR, Gurler O, Birlik M, et al. Hyperuricemia and its related factors in an urban population, Izmir, Turkey. Rheumatol Int. 2009; 29:869-74.

126. Zhu Y, Pandya BJ, Choi HK. Prevalence of gout and hyperuricemia in the US general population: the national health and nutrition examination survey 2007-2008. Arthritis Rheum. 2011;63:3136-41.

127. Zhang L, Wang F, Wang $L$, et al. Prevalence of chronic kidney disease in China: a cross-sectional survey. Lancet. 2012;379:815-22.

128. Song $P$, Wang $H$, Xia W, Chang $X$, Wang $M, A n L$. Prevalence and correlates of hyperuricemia in the middle-aged and older adults in China. Sci Rep. 2018:8:4314.

129. Rho YH, Zhu Y, Choi HK. The epidemiology of uric acid and fructose. Semin Nephrol. 2011;31:410-9.

130. Ghei M, Mihailescu M, Levinson D. Pathogenesis of hyperuricemia: recent advances. Curr Rheumatol Rep. 2002;4:270-4.

131. Kumar S, Singh A, Takhelmayum R, Shrestha P, Sinha J. Prevalence of hyperuricemia in Chitwan District of Nepal. J Coll Med Sci Nepal. 2010;6:18-23.

132. Jularattanaporn V, Krittayaphong R, Boonyasirinant T, Udol K, Udompunurak S. Prevalence of hyperuricemia in Thai patients with acute coronary syndrome. Thai Heart J. 2008;21:86-92.

133. Al-Arfaj AS. Hyperuricemia in Saudi Arabia. Rheumatol Int. 2001;20:61-4.

134. Conen D, Wietlisbach V, Bovet $\mathrm{P}$, et al. Prevalence of hyperuricemia and relation of serum uric acid with cardiovascular risk factors in a developing country. BMC Public Health. 2004;4:9.

135. Klemp P, Stansfield SA, Castle B, Robertson MC. Gout is on the increase in New Zealand. Ann Rheum Dis. 1997:56:22-6.

136. Pan B, Zhang Q, Zhou H, Ma ZF. Prevalence of components of metabolic syndrome among adults with the presence of autoimmune thyroid condition in an iodine-sufficient region. Biol Trace Elem Res. 2020. https:// doi.org/10.1007/s12011-020-02413-3.

Ready to submit your research? Choose BMC and benefit from:

- fast, convenient online submission

- thorough peer review by experienced researchers in your field

- rapid publication on acceptance

- support for research data, including large and complex data types

- gold Open Access which fosters wider collaboration and increased citations

- maximum visibility for your research: over $100 \mathrm{M}$ website views per year

At $\mathrm{BMC}$, research is always in progress.

Learn more biomedcentral.com/submissions 